\title{
The effect of compressibility on turbulent shear flow: a rapid-distortion-theory and direct-numerical-simulation study
}

\author{
By A. SIMONE 1 , G. N. COLEMAN ${ }^{2}$ AND C. CAMBON \\ ${ }^{1}$ Ecole Centrale de Lyon, Laboratoire de Mécanique des Fluides et d'Acoustique, \\ UMR CNRS $n^{0} 5509,69131$ Ecully cedex, France \\ ${ }^{2}$ University of California, Los Angeles, Mechanical and Aerospace Engineering Department, \\ 48-121 Engr. IV, Box 951597, Los Angeles, CA 90095-1597, USA
}

(Received 4 July 1996)

The influence of compressibility upon the structure of homogeneous sheared turbulence is investigated. For the case in which the rate of shear is much larger than the rate of nonlinear interactions of the turbulence, the modification caused by compressibility to the amplification of turbulent kinetic energy by the mean shear is found to be primarily reflected in pressure-strain correlations and related to the anisotropy of the Reynolds stress tensor, rather than in explicit dilatational terms such as the pressuredilatation correlation or the dilatational dissipation. The central role of a 'distortion Mach number' $M_{d}=S \ell / a$, where $S$ is the mean strain or shear rate, $\ell$ a lengthscale of energetic structures, and $a$ the sonic speed, is demonstrated. This parameter has appeared in previous rapid-distortion-theory (RDT) and direct-numerical-simulation (DNS) studies; in order to generalize the previous analyses, the quasi-isentropic compressible RDT equations are numerically solved for homogeneous turbulence subjected to spherical (isotropic) compression, one-dimensional (axial) compression and pure shear. For pure-shear flow at finite Mach number, the RDT results display qualitatively different behaviour at large and small non-dimensional times $S t$ : when $S t<4$ the kinetic energy growth rate increases as the distortion Mach number increases; for $S t>4$ the inverse occurs, which is consistent with the frequently observed tendency for compressibility to stabilize a turbulent shear flow. This 'crossover' behaviour, which is not present when the mean distortion is irrotational, is due to the kinematic distortion and the mean-shear-induced linear coupling of the dilatational and solenoidal fields. The relevance of the RDT is illustrated by comparison to the recent DNS results of Sarkar (1995), as well as new DNS data, both of which were obtained by solving the fully nonlinear compressible Navier-Stokes equations. The linear quasi-isentropic RDT and nonlinear non-isentropic DNS solutions are in good general agreement over a wide range of parameters; this agreement gives new insight into the stabilizing and destabilizing effects of compressibility, and reveals the extent to which linear processes are responsible for modifying the structure of compressible turbulence.

\section{Introduction}

There is now consensus in the literature that the 'intrinsic compressibility' (nonzero divergence) of a turbulent velocity field tends to inhibit mixing and reduce the 
amplification rate of turbulent kinetic energy produced by a mean velocity gradient, with respect to the purely solenoidal case. This has been observed, for example, in experimental (Papamoschou \& Roshko 1988; Clemens \& Mungal 1995) and numerical (Sandham \& Reynolds 1991; Vreman, Sandham \& Luo 1996) studies of mixing layers, and has been demonstrated by direct numerical simulation (DNS) of compressible homogeneous shear flow (Blaisdell, Mansour \& Reynolds 1991, 1993; Sarkar, Erlebacher \& Hussaini 1991a; Sarkar 1995). (The reader is referred to Lele 1994 for an overview of other numerical investigations; the experimental work is surveyed in Fernholz \& Finley 1977, 1980, Fernholz et al. 1989 and Settles \& Dodson 1991. See Bradshaw 1977, Friedrich 1993, Lele 1994 and Spina, Smits \& Robinson 1994 for general introductions to compressible turbulent flows.) On the other hand, recent analyses by Jacquin, Cambon \& Blin (1993) and Cambon, Coleman \& Mansour (1993) (see also Lele 1994) have shown that, because of the multi-timescale and initial-value nature of the problem, the compressibility-induced growth-rate reduction is not universal. They used rapid distortion theory (RDT) and DNS to show that when a sufficiently broad parameter range is considered, the flow can experience an increase with increasing compressibility (as measured by a relevant Mach number) of the kinetic energy growth rate when the turbulence is subjected to rapid (but finite) axial compression. Since the RDT analysis is valid for mean deformations other than axial compression (see Cambon et al. 1993 and below), it is possible that under certain conditions homogeneous shear flow might also fail to exhibit a decrease of the kinetic energy amplification rate with increasing Mach number, and thus fail to display the behaviour commonly thought to be typical of compressible turbulence. Such conditions do in fact exist, as this paper will show.

Both analytical and numerical studies reveal the relevance of a 'distortion Mach number' $M_{d}=S \ell / a$, to parameterize rapidly sheared or strained compressible turbulence; here $S$ is a mean strain or shear rate, $\ell$ an integral lengthscale and $a$ the mean sonic speed. This parameter was first introduced by Durbin \& Zeman (1992), and denoted ' $\Delta M$ ', since it can be interpreted as the mean Mach number change across an 'eddy'. Durbin \& Zeman's RDT analysis only addressed irrotational deformations and is restricted to small values of $\Delta M$. Jacquin et al. (1993) and Cambon et al. (1993) later investigated the full $\Delta M$ range for the irrotationally strained case; they defined the solenoidal and 'pressure-released' regimes, associated with the small and large $\Delta M$ limits, respectively, and using RDT and DNS found a monotonic increase of the turbulent energy growth rate with increasing $\Delta M$. Because $\Delta M$ can be interpreted as the product of a turbulent Mach number $M_{t}$ and the ratio $r$ of turbulent to mean-distortion timescales (see below), an increase of $\Delta M$ represents, for fixed $r$, an increase of $M_{t}$; this monotonic increase in growth rate observed when passing from the solenoidal to pressure-released regime can therefore be viewed as an example of the 'atypical' compressible-turbulence behaviour cited above (since an increase in $M_{t}$ is not associated with increased stability). Sarkar (1995) also used the $S \ell / a$ parameter (which he referred to as a 'gradient Mach number' $M_{g}$ ), to quantify compressibility effects for homogeneous shear flow. But instead of agreeing with the axial-strain findings, his DNS results show, at large non-dimensional times $S t$, a monotonic decrease of the turbulent kinetic energy growth rate with increasing $M_{g}$, consistent with the usual understanding that compressibility tends to stabilize the turbulence. At first glance, the lack of destabilization with increasing $M_{g}$ might be ascribed to the irrelevance of the rapid-distortion limit to the shear-flow runs he studied, which would in turn imply that linear processes play no role in the $M_{g}$-dependence he observed. The recent work of Simone \& Cambon $(1995 a, b)$, however, suggests that this is not 
the case: as we will show below, the destabilizing influence as $M_{g} \rightarrow \infty$ (including the range considered by Sarkar) is also present for pure-shear distortions, but only at small $S t$; for large $S t$ the analysis predicts the classic stabilization with increasing $M_{g}$ found by Sarkar (1995). Note that because the axially compressed case can only exist for finite times (the time-dependent strain rate is given by $S(t)=S_{0} /\left(1+S_{0} t\right)$, where $S_{0}=S(0)$ is the initial rate of compression - and thus at $\left|S_{0}\right| t=1$ the flow domain collapses to a single point; Cambon et al. 1993), there was no need in the previous axial-compression study to differentiate between long- and short-time behaviour in terms of $\left|S_{0}\right| t$. Interestingly, Sarkar's (1995) results also exhibit trends with $M_{g}$ that are opposite at large and small $S t$, and therefore consistent with the findings presented here (see his figure 1, for example). The early-time behaviour was not discussed, however, since he focused on asymptotic $M_{g}$-dependence at large $S t$, and could therefore use 'unphysical' initial conditions, thus producing data at small $S t$ that are not fully representative of a 'real' turbulent field. (This is appropriate because the flow 'forgets' its initial state under the influence of the mean shear, and becomes valid at later times (Blaisdell et al. 1993; Sarkar 1995).) In the DNS data shown here, any early-time ambiguity is avoided by 'aging' the initial conditions until they become 'physical', as explained below.

The parameter $\Delta M \sim M_{g} \sim S \ell / a$ appears naturally when scaling the (linearized) RDT equations for homogeneous compressible turbulence. In view of this generality, this parameter will henceforth be solely referred to as the 'distortion Mach number' $M_{d}$ (following a suggestion by L. Jacquin, private communication). The separate relevance of both a 'distortion' and a 'turbulent' Mach number (Sarkar 1995) indicates the previously mentioned multi-timescale nature of the problem: at least three different timescales must be considered. These are the mean distortion time,

$$
\tau_{d}=\left(U_{i, j} U_{i, j}\right)^{-1 / 2},
$$

the 'turbulent decay' or 'turn-over' time,

$$
\tau_{t}=\ell / q,
$$

and the timescale linked to the sonic speed,

$$
\tau_{a}=\ell / a
$$

(where $U_{i, j}$ is the mean velocity gradient, and $\frac{1}{2} q^{2}$ is the turbulent kinetic energy). The distortion speed, $r=\tau_{t} / \tau_{d}$, is the only parameter needed to characterize homogeneous turbulence that is incompressible, at least for large Reynolds numbers. However, when intrinsic compressibility is present, the ratio of the two latter timescales, which amounts to a turbulent Mach number $M_{t}=\tau_{a} / \tau_{t}=q / a$, must also be accounted for. A third non-dimensional ratio, the distortion Mach number $M_{d}=\tau_{a} / \tau_{d}$, is also relevant. Any two of these three parameters (along with the ratio of specific heats, the Reynolds and Prandtl numbers, and possibly the initial conditions) uniquely defines the state of the compressible flow, although Sarkar (1995) has shown that compressibility effects are more sensitive to variations in $M_{d}$ than they are to changes in $M_{t}$. The reasons for the crucial role of $M_{d}$ will be revealed below.

Two approaches have previously been taken when attempting to determine the effects of compressibility on turbulence. The first, which we shall refer to as the 'explicit' or 'energetic' approach, involves measuring the dilatational terms (the pressuredilatation and dilatational-dissipation correlations) that appear in the turbulent 
kinetic energy (TKE) budget. For homogeneous turbulence, this equation is

$$
\frac{\mathscr{D}}{\mathscr{D} t}\left(\frac{q^{2}}{2}\right)=\mathscr{P}-\epsilon_{s}-\epsilon_{d}+\Pi_{d},
$$

in which $\mathscr{P}$ is the rate of production by the mean flow, $\epsilon_{s}$ the rate of solenoidal dissipation, $\epsilon_{d}$ the rate of dilatational dissipation, and $\Pi_{d}$ the pressure-dilatation correlation (Blaisdell et al. 1991). Note that the last two terms on the right-hand side do not appear when the flow is incompressible. The explicit/energetic approach is embodied in the modelling of $\epsilon_{d}$ and $\Pi_{d}$ done by Zeman (1990), Sarkar et al. (1991b) and others.

On the other hand, one may use an 'implicit' strategy (as is done here) whereby the effects of compressibility are assumed to be felt primarily through modification to the structure of the turbulence - which affects the pressure field, the pressure-strain correlation tensor, the anisotropy of the velocity field, and in turn the production term $\mathscr{P}$ in the TKE equation. Recall that $\mathscr{P}$ depends on the anisotropy of the Reynolds-stress tensor (RST) since $\mathscr{P}=-\overline{u_{i} u_{j}} U_{i, j}$ with $\overline{u_{i} u_{j}}=q^{2}\left(\delta_{i j} / 3+b_{i j}\right)$, and that $b_{i j}$, the Reynolds-stress anisotropy tensor, is governed by an equation that involves the pressure-strain tensor. The recent homogeneous-shear DNS results of Sarkar (1995), cited above, demonstrate the relevance of the implicit/structural approach, since the effect of compressibility was found to modify the RST anisotropy component $b_{12}$ much more significantly than it does the explicit dilatational terms. Moreover, Speziale, Abid \& Mansour (1995) have used the DNS results of Blaisdell et al. (1993) to find that for homogeneous shear all components of $b_{i j}$ are altered by compressibility. Any RDT study naturally accommodates the implicit/structural method, since it can provide a straightforward prediction of the 'rapid part' of the fluctuating component of pressure and the related pressure-strain correlations, as well as changes to $b_{i j}$. In contrast, Durbin \& Zeman (1992) used RDT to illustrate the role, during a mean axial compression with low $M_{d}$, of the explicit contribution provided by the pressure-dilatation term, and proposed that it should be taken into account when $M_{t} \approx 0$ and $r \gg 1$. Although $\Pi_{d}$ is likely to be important in some situations (in shock-turbulence interactions, for example), its significance was perhaps unrealistically magnified in their study, since it was measured with respect to the rate of TKE dissipation; when compared to the rate of TKE production - the dominant term during a rapid compression - it appears much less important. A recent DNS study of the compressible mixing layer (Vreman et al. 1996) has shown that the pressure-dilatation and dilatational-dissipation terms are insignificant, even when the convective Mach number is of order one. This also points to the implicit, rather than the explicit, as the more relevant of the two approaches.

As with efforts to close the TKE equation, attempts to construct second-order models valid for fully compressible flows began, quite naturally, with the explicit/energetic approach; unfortunately (as one might expect from the above survey), these led to a tendency to over-emphasize the role of explicit dilatational terms (see also Huang, Coleman \& Bradshaw 1995 for more on this point); little attention has been paid to modifications to pressure-strain correlations and the related anisotropic structure of the RST (see Cambon et al. 1993 for an exception).

The aim of this paper is to extend the rapid distortion analysis previously applied to irrotationally strained flow to compressible homogeneous turbulence subjected to pure shear. It thus extends the work of Cambon et al. (1993) and completes the study begun by Simone \& Cambon $(1995 a, b)$. The degree to which RDT can 
be used to explain previously observed results, especially those of Sarkar (1995), is ascertained. We find that many of the features of compressible shear flow that were previously thought to be solely due to nonlinear processes (in particular, the stabilization associated with intrinsic compressibility) are captured by the RDT and thus are due to modifications governed by linear equations. These linearized equations are solved for the parameters of interest using a generalized RDT code (Simone 1995). In order to determine the importance of nonlinear and non-isentropic effects, the RDT results are compared to full Navier-Stokes solutions obtained via DNS, using the code of Spyropoulos \& Blaisdell (1996).

The paper is organized as follows. The formalism of compressible quasi-isentropic RDT is introduced in $\$ 2$, with a brief overview of the numerical method given. Two test cases (isotropic and axial compression) are presented in $\S 3$ to validate the RDT code; results are contrasted to analytical solutions when they exist, and to appropriate DNS data when they do not. A RDT and DNS study of compressible homogeneous turbulence subjected to pure shear is discussed in $\$ 4$. A summary and discussion of the main results and conclusions are contained in $\S 5$, which also revisits the issue of the stabilizing effect of compressibility in turbulent shear flow.

\section{Rapid-distortion analysis for compressible homogeneous turbulence}

Rapid distortion theory (Batchelor \& Proudman 1954) combines linearization of the governing equations with averaging to describe histories of the statistics of turbulence in the presence of mean deformations that are rapid compared to timescales of the fluctuations. The starting points of RDT and linear stability analysis are the same, provided the background field defined in the RDT is a solution of the Euler equations, and that the disturbances are statistically homogeneous. A convenience of the RDT technique is that solutions of the linearized equations can be computed a priori, before they are used to determine the evolution of the statistics: the linear 'transfer matrix' that links any realization of the disturbance field at time $t$ to its initial state at $t=0$ can be computed independently of the disturbance field, in terms of the initial wavevector, the mean-flow characteristics and the elapsed time. This matrix, denoted $\boldsymbol{g}$ in the following, contains all the information of a linear stability analysis, and as shown below allows the history of the statistics to be obtained once the initial spectrum tensors are specified.

It is generally assumed that linear solutions are valid for turbulent flows only for distortions that are sufficiently rapid (such that $S \tau_{t}=S \ell / q \gg 1$ ) and limited to short times (compared to $S t$ ) and large scale (or small wavenumber $k$, with $k q / S \ll 1$ ). In practice, however, the exact range of validity is difficult to predict, even for the puresolenoidal case. Analytical investigations of the relative importance of the nonlinear and linear terms are just beginning (for example Kevlahan \& Hunt 1996, who consider irrotational distortions of solenoidal turbulence). These studies are complicated by a number of factors, including the narrow domain in spectral space of the relevant (most amplified) modes and the temporal distortion of the wavevectors. We stress that 'global' parameters that use single characteristic scales (such as $S \ell / q$ ) to indicate the relevance of the rapid-distortion solutions can only be approximate, and in fact the predictive success of such measures will depend upon the statistical quantity being examined. Consequently, the extent to which the RDT predictions will agree with the actual (in this case, DNS) results will only be fully known after the two have been compared. After the flow parameters have been specified, and the comparison has 
yielded this a posteriori information about the importance of nonlinear mechanisms, the practical significance of this study should be clear.

In compressible turbulence, the disturbance field includes solenoidal and dilatational velocity modes, and an entropic mode. In the linear inviscid limit, and in the absence of mean gradients or body forces, the dilatational velocity is coupled to pressure disturbances that satisfy an acoustic wave equation, and the solenoidal (or vortical), acoustic and entropic modes are independent of each other (Kovasznay 1953; Monin \& Yaglom 1971), and, for example, the pressure fluctuations have no influence on the solenoidal component. Nonlinear interactions in weakly compressible homogeneous isotropic turbulence (i.e. with no mean gradients) were studied by Marion (1988) using a two-point closure model. He showed that a solenoidal field can generate and sustain an acoustic field through nonlinear interactions (the entropic mode was removed from consideration by making the barotropic assumption). In the compressible RDT of Durbin \& Zeman (1992), Jacquin et al. (1993) and Cambon et al. (1993), the turbulence is assumed to be quasi-isentropic, in that entropy fluctuations are only advected by the mean flow - which implies only equations for velocity and pressure disturbances need to be considered, as in the Marion (1988) study. But since the linearized equations can become strongly coupled due to the mean velocity gradient when the flow is subjected to a mean deformation, the acoustic regime can be modified and even disappear for high values of the distortion Mach number $M_{d}$. This will be illustrated shortly.

Assuming that the fluid is an ideal gas with constant specific heats, and that the fluctuations of density $\rho^{\prime}$ are much smaller than their mean $\bar{\rho}$, the equations for the fluctuating (disturbance) component of velocity $u_{i}$, pressure $p$ and entropy $s$ can be written

$$
\begin{aligned}
\dot{u}_{i}+U_{i, j} u_{j} & =-\frac{p_{, i}}{\bar{\rho}}, \\
\left(\frac{\dot{p}}{\gamma P}\right) & =-u_{i, i}, \\
\dot{s} & =0,
\end{aligned}
$$

where lower- and upper-case italic symbols will be used throughout to denote respectively fluctuation and mean (Reynolds-averaged) quantities; $\gamma$ is the ratio of specific heats. Viscous terms are omitted for now for the sake of brevity (they will briefly re-enter the analysis below). Employing the formalism of continuum mechanics (see e.g. Eringen 1980), spatial derivatives in (2.1) are with respect to the Eulerian (spatial) coordinate $x_{i}$, and the dot superscript denotes a substantial derivative along mean flow trajectories, which is the partial derivative with respect to time at fixed Lagrangian (material) coordinate $X_{i}$, defined by

$$
\text { ( ) }=\frac{\partial()}{\partial t}+U_{i}()_{, i} \text {. }
$$

The relationship between the Eulerian and Lagrangian coordinates is given by

$$
x_{i}=x_{i}(\boldsymbol{X}, t, 0) \quad \text { and } \quad \mathrm{d} x_{i}=U_{i} \mathrm{~d} t+F_{i j} \mathrm{~d} X_{j},
$$

where $x_{i}$ is the position at time $t$ of a fluid particle moving with the mean flow, which has the position $X_{i}$ at the initial time $t=0$ (hence the third argument for $\boldsymbol{x}$ in (2.3)), and $U_{i}=\dot{x}_{i}=\left(\partial x_{i} / \partial t\right)_{X}$, the latter derivative being at fixed $\boldsymbol{X}$. The gradient displacement tensor $F_{i j}=\partial x_{i} / \partial X_{j}$ (Eringen 1980) is defined by the mean-flow distortion. Limiting ourselves to mean flows that maintain the spatial homogeneity of the turbulence requires the mean pressure gradient $\partial P / \partial x_{i}$ to be zero 
and the mean velocity gradients $U_{i, j}$ to be uniform in space (Blaisdell et al. 1991). Therefore

$$
U_{i}=U_{i, j}(t) x_{j}
$$

and

$$
\dot{U}_{i}=\left(\dot{U}_{i, j}+U_{l, j} U_{i, l}\right) x_{j}=X_{j} \ddot{F}_{i j}=0,
$$

where we have used

$$
\dot{F}_{i j}=U_{i, l} F_{l j} \quad \text { and } \quad x_{i}=F_{i j}(t, 0) X_{j},
$$

which is the solution of $\dot{x}_{i}=U_{i, j} x_{j}$ with $x_{i}=X_{i}$ at $t=0$, and specifies (2.3) for space-uniform distortions. This implies (Cambon et al. 1993)

$$
F_{i j}(t, 0)=\delta_{i j}+A_{i j} t \quad \text { and } \quad U_{i, j}(t)=A_{i l} F_{l j}^{-1}(t, 0),
$$

where $\boldsymbol{F}^{-1}$ (which is equivalent to ' $\boldsymbol{B}$ ' in Rogallo 1981) is the inverse of $\boldsymbol{F}$. The above equation describes the most general homogeneity-preserving mean flow, for which the mean acceleration $\dot{U}_{i}=\ddot{x}_{i}=\ddot{F}_{i j} X_{j}$ is zero; specific examples for various distortions are given by Blaisdell et al. (1991). Equation (2.7) is valid for an arbitrary constant (not necessarily symmetric) matrix $\boldsymbol{A}$, provided the determinant of $\boldsymbol{F}$, which is equal to the volumetric ratio $J(t)=\bar{\rho}(0) / \bar{\rho}(t)$, remains positive.

The Helmholtz decomposition of the velocity field and the subsequent solution of (2.1) are facilitated by using a three-dimensional Fourier transform (denoted here by the symbol $\left.{ }^{`}\right)$. The Fourier transform $\widehat{u}_{i}(\boldsymbol{k}, t)$ of the velocity fluctuation $u_{i}$ is expressed in an orthonormal frame of reference with bases $\left(\boldsymbol{e}^{(1)}, \boldsymbol{e}^{(2)}, \boldsymbol{e}^{(3)}\right)$ normal and parallel to the the wavevector $\boldsymbol{k}$, with the parallel component $e_{i}^{(3)}=k_{i} / k$, where $k$ is the wavevector modulus. As a result

$$
\widehat{u}_{i}(\boldsymbol{k}, t)=\underbrace{\widehat{\varphi}^{(1)}(\boldsymbol{k}, t) e_{i}^{(1)}(\boldsymbol{k})+\widehat{\varphi}^{(2)}(\boldsymbol{k}, t) e_{i}^{(2)}(\boldsymbol{k})}_{\widehat{u_{i}^{s}}}+\underbrace{\widehat{\varphi}^{(3)}(\boldsymbol{k}, t) e_{i}^{(3)}(\boldsymbol{k})}_{\widehat{u}_{i}^{d}} .
$$

The first two terms are the Fourier transform of the solenoidal velocity $\widehat{u}_{i}^{s}$; the third is the dilatational contribution $\widehat{u}_{i}^{d}$. This representation involves the minimum number of necessary independent components and conserves all tensoral properties (invariants) of the velocity field. Note that the solenoidal bases $\left(\boldsymbol{e}^{(1)}, \boldsymbol{e}^{(2)}\right)$ are specified to within an angle of rotation around the wavevector $\boldsymbol{k}$; a more precise definition (see Cambon et al. 1993) will be used when needed. Classical descriptions in terms of vorticity $\omega_{i}$ and divergence $u_{i, i}$ are easily recovered as

$$
\widehat{\omega}_{i}=\mathrm{i} k\left(\widehat{\varphi}^{(1)} e_{i}^{(2)}-\widehat{\varphi}^{(2)} e_{i}^{(1)}\right)
$$

and

$$
\widehat{u}_{i, i}=\mathrm{i} k \widehat{\varphi}^{(3)} .
$$

For convenience, the pressure mode is non-dimensionalized by the mean sound speed $a=(P / \bar{\rho})^{1 / 2}$, so that the fourth dependent variable becomes

$$
\widehat{\varphi}^{(4)}=\mathrm{i} \frac{\widehat{p}}{\bar{\rho} a} .
$$

A linear system of equations for $\widehat{\varphi}^{(i)}$, with $i=(1,2,3,4)$, can now be derived from 
(2.1):

$$
\begin{aligned}
\dot{\hat{\varphi}}^{(\alpha)}-\left(U_{l, l}-v k^{2}\right) \widehat{\varphi}^{(\alpha)}+m_{\alpha \beta} \widehat{\varphi}^{(\beta)}+m_{\alpha 3} \widehat{\varphi}^{(3)} & =0, \\
\dot{\hat{\varphi}}^{(3)}-\left(U_{l, l}-\frac{4}{3} v k^{2}-m_{33}\right) \widehat{\varphi}^{(3)}+m_{3 \alpha} \widehat{\varphi}^{(\alpha)}+k a \widehat{\varphi}^{(4)} & =0, \\
\dot{\hat{\varphi}}^{(4)}-\frac{1}{2}(3-\gamma) U_{l, l} \widehat{\varphi}^{(4)}-k a \widehat{\varphi}^{(3)} & =0 .
\end{aligned}
$$

The operator ( ) $-U_{l, l}()$ is the Fourier transform of the substantial derivative (where $U_{l, l}=U_{1,1}+U_{2,2}+U_{3,3}$ is the mean divergence), $v$ is the kinematic viscosity and the $m_{i j}$ components are given by

$$
\begin{aligned}
& m_{\alpha \beta}=e_{i}^{(\alpha)} U_{i, j} e_{j}^{(\beta)}-\dot{e}_{i}^{(\alpha)} e_{i}^{(\beta)}=e_{i}^{(\alpha)} U_{i, j} e_{j}^{(\beta)}-\epsilon_{\alpha \beta 3} R_{E}, \\
& m_{\alpha 3}=e_{i}^{(\alpha)} U_{i, j} e_{j}^{(3)}-\dot{e}_{i}^{(\alpha)} e_{i}^{(3)}=e_{i}^{(\alpha)}\left(U_{i, j}-U_{j, i}\right) e_{j}^{(3)}, \\
& m_{3 \alpha}=e_{i}^{(3)} U_{i, j} e_{j}^{(\alpha)}-\dot{e}_{i}^{(3)} e_{i}^{(\alpha)}=2 e_{i}^{(3)} U_{i, j} e_{j}^{(\alpha)}, \\
& m_{33}=e_{i}^{(3)} U_{i, j} e_{j}^{(3)},
\end{aligned}
$$

where Greek super/subscripts (indicating solenoidal space) take only the value 1 or 2. The viscous terms in (2.12) have been included for the sake of completeness, but are henceforth neglected (see Simone 1995 for a discussion of their influence). The 'rotation term' $R_{E}$ in $(2.13 a)$ is $e_{i}^{(2)} U_{i, j} e_{j}^{(1)}$ if the polar axis is chosen as one of the eigenvectors of the mean gradient tensor; its general expression is available in Cambon, Teissedre \& Jeandel (1985). The overdot denotes a time derivative at fixed $\boldsymbol{K}$, which plays the same role here as $\boldsymbol{X}$ does in physical space, since

$$
k_{i} x_{i}=K_{i} X_{i} \quad \text { with } \quad k_{i}=F_{j i}^{-1}(t, 0) K_{j} .
$$

This reveals how the distortions in spectral and physical space are linked, and displays an advantage of using the 'mean-trajectories' formalism. In the absence of viscosity, solutions of $(2.12 a)-(2.12 c)$ can be expressed as

$$
\widehat{\varphi}^{(i)}(\boldsymbol{k}, t)=J(t) g_{i j}(\boldsymbol{k}, t, 0) \widehat{\varphi}^{(j)}(\boldsymbol{K}, 0),
$$

where $\widehat{\varphi}^{(j)}(\boldsymbol{K}, 0)$ are the initial values of the velocity and pressure modes, and $g_{i j}$ are elements of the linear transfer matrix, which depend in general on both the direction and magnitude of the wavevector $\boldsymbol{k}$, and implicitly on time and mean compression (if the mean velocity gradient has a non-zero trace). The volumetric ratio $J(t)=\bar{\rho}(0) / \bar{\rho}(t)$ in $(2.15)$ accounts for the explicit mean compression (dilatation) term present in $(2.12)$. Once $g_{i j}(t)$ for $i, j=(1,2,3,4)$ are known, they can be used to construct RDT histories of relevant covariance matrices, such as the second-order spectral tensors, and then to compute single-point correlations by integrating over the three-dimensional wavespace. To obtain solutions of $\boldsymbol{g},(2.15)$ is substituted into $(2.12 a)-(2.12 c)$, which is then solved numerically using a matrix exponentiation method for an arbitrary set of initial conditions such that $\widehat{\varphi}^{(i)}=\left(\delta_{i 1}, \ldots, \delta_{i 4}\right)$. The spectrum-tensor solutions $\left\langle\widehat{\varphi}^{(i)^{*}}(\boldsymbol{p}, t) \widehat{\varphi}^{(j)}(\boldsymbol{k}, t)\right\rangle$ are then formed, using $(2.15)$, as products of $\boldsymbol{g}$ and appropriate initial spectra, which are derived by assuming that the fields are initially isotropic and in 'acoustic equilibrium' (see below).

Initially isotropic, second-order correlations are given by

$$
\left\langle\widehat{\varphi}^{(i) *}(\boldsymbol{P}, 0) \widehat{\varphi}^{(j)}(\boldsymbol{K}, 0)\right\rangle=\frac{E^{(i i)}(K, 0)}{2 \pi K^{2}} \delta_{i j} \delta(\boldsymbol{K}-\boldsymbol{P}) ; \quad i, j=(1,2,3,4)
$$

(no sum over $i$ ), where $E^{(11)}=E^{(22)}=\frac{1}{2} E^{(s)}, E^{(33)}=E^{(d)}, E^{(44)}=E^{(p)}$, and $E^{(s)}$, 
$E^{(d)}$, and $E^{(p)}$ are respectively the conventional solenoidal, dilatational and 'pressure' (or 'potential') energy spectra; the asterisk denotes a complex conjugate, $\delta$ is the Dirac delta, and the angle brackets represent an ensemble average over statistically independent realizations of the initial field. Note that because any second-order statistical tensor can be written as the product of a deterministic quantity (quadratic in $\boldsymbol{g}$ ) and initial (ensemble-averaged) spectra $E^{(i i)}(K, 0)$, only the initial spectra - and not the individual realizations (e.g. $\widehat{\varphi}^{(i)}$ and $\widehat{\varphi}^{(j)}$ ) that make up the ensemble average in (2.16) - must be specified to complete the initialization. For the sake of simplicity, the initial fields are assumed to be in a state of 'acoustic equilibrium' (Sarkar et al. 1991b); this amounts to setting the kinetic energy of the dilatational mode equal to the potential energy of pressure mode, such that $\frac{1}{2}\left\langle\left(\varphi^{(3)}\right)^{2}\right\rangle$ and $\frac{1}{2}\left\langle p^{2}\right\rangle /(\bar{\rho} a)^{2}$ are identical. A 'strong form' of this relation is employed here, by assuming that the balance occurs at each wavenumber of the initial pressure and dilatational-velocity spectra, with $E^{(d)}=E^{(p)}$ in (2.16).

Further details regarding (2.12) are found for the inviscid case in Cambon et al. (1993). For present purposes we note that the matrix $m_{i j}$ has dimensions of an inverse timescale $\tau^{-1}$, which in general is determined by the relationship between the magnitude of the mean gradient $S=\left|U_{i, j}\right|$ and the sonic scale $a k$ (and the viscous terms; see Simone 1995). The RDT equations thus clearly display the importance of the ratio of the gradient and sonic scales, $S / a k$, which at wavenumbers representing the large energetic eddies is proportional to $M_{d}$. More insight into the equation set results when the variables

$$
y=\widehat{\varphi}^{(3)} / J k \quad \text { and } \quad z=\widehat{\varphi}^{(4)} / J a=\mathrm{i} \widehat{p} / J \bar{\rho} a^{2}
$$

are introduced; they allow (2.12) to be simplified to

$$
\begin{aligned}
& \frac{\mathscr{D}}{\mathscr{D} t}\left(\frac{\dot{y}}{a^{2}}\right)+k^{2} y=-\dot{z}^{s}, \\
& \frac{\mathscr{D}}{\mathscr{D} t}\left(\frac{\dot{z}}{k^{2}}\right)+a^{2} z=+a^{2} z^{s},
\end{aligned}
$$

where the solenoidal pressure term is

$$
z^{s}=\frac{m_{3 \alpha} \widehat{\varphi}^{(\alpha)}}{J k a^{2}}=\mathrm{i} \frac{\widehat{p}_{s}}{J \bar{\rho} a^{2}},
$$

with $\widehat{p}_{s} / \bar{\rho}=m_{3 \alpha} \widehat{\varphi}^{(\alpha)} / k$ (sum over $\alpha$ ); for the sake of clarity, $\mathscr{D}() / \mathscr{D} t$ has been used in addition to the overdot notation to represent the substantial derivative. This form of the equations immediately illustrates the relevant flow regimes. The incompressible (solenoidal) limit is recovered when $a \rightarrow \infty$, and (2.19) reduces to $z=z^{s}$ (which corresponds to the Poisson equation for the fluctuating pressure). Two distinct non-solenoidal regimes can also be discerned, by considering the relative magnitude of the terms in (2.18). When $\dot{z}^{s}$ is negligible, oscillating acoustic behaviour is found, corresponding to Kovasznay's (1953) state of uncoupled vortical, acoustic and entropic modes. This is the state usually assumed by other RDT calculations of distorted compressible turbulence (e.g. Lee, Lele \& Moin 1993). The current RDT can account for more general cases, for which the solenoidal and dilational modes are strongly coupled; an example is the pressure-released limit discussed by Jacquin et al. (1993) and Cambon et al. (1993), which is recovered when $k^{2} y$ is ignored (since this term couples the pressure and velocity fields: note that in terms of the modified variables, the pressure transport equation $(2.1 b)$ is $\dot{z}=k^{2} y$ ). The role of the distortion 
Mach number in defining the pressure-released limit becomes clear when the initial values $S_{0}^{-1}, k_{0}^{-1}$, and $a_{0}$ are used as characteristic time, length and velocity scales, respectively, to define the non-dimensional variables (cf. Jacquin et al. 1993)

$$
\widetilde{t}=S_{0} t ; \quad \widetilde{k}=\frac{k}{k_{0}} ; \quad \widetilde{a}=\frac{a}{a_{0}} ; \quad \widetilde{y}=\frac{k_{0} y}{a_{0}} ; \quad \widetilde{\varphi}^{(3)}=\frac{\widehat{\varphi}^{(3)}}{a_{0}} ; \quad \widetilde{z}^{s}=\frac{k_{0} a_{0} z^{s}}{S_{0}} .
$$

Equation (2.18) can thus be written

$$
\frac{\mathscr{D}}{\mathscr{D} \tilde{t}}\left(\frac{1}{\widetilde{a}^{2}} \frac{\mathscr{D} \widetilde{y}}{\mathscr{D}}\right)+\frac{\widetilde{k}^{2} \widetilde{y}}{\mathscr{M}_{d}^{2}}=-\frac{1}{\mathscr{M}_{d}} \frac{\mathscr{D} z^{s}}{\mathscr{D} \tilde{t}}=-\frac{\mathscr{D} \widetilde{z}^{s}}{\mathscr{D} \widetilde{t}},
$$

which displays the significance of the distortion Mach number $M_{d} \sim \mathscr{M}_{d}=S_{0} / k_{0} a_{0}$, in addition to the irrelevance as $M_{d} \rightarrow \infty$ of the $\widetilde{k}^{2} \widetilde{y}$ pressure-source term associated with the pressure-released limit. To obtain the non-dimensional form appropriate for the $M_{d} \rightarrow 0$ limit, one must use $\left(a_{0} k_{0}\right)^{-1}$ rather than $S_{0}^{-1}$ as the characteristic time, yielding for the $M_{d} \rightarrow 0$ flow

$$
\frac{\mathscr{D}}{\mathscr{D} \widetilde{t}}\left(\frac{1}{\widetilde{a}^{2}} \frac{\mathscr{D} \widetilde{y}}{\mathscr{D}}\right)+\widetilde{k}^{2} \widetilde{y}=-\frac{\mathscr{D} z^{s}}{\mathscr{D} \widetilde{t}}=-\mathscr{M}_{d} \frac{\mathscr{D} \widetilde{z}^{s}}{\mathscr{D} \widetilde{t}}
$$

where now $\widetilde{t}=a_{0} k_{0} t$. This shows that the 'uncoupled-mode' state mentioned above, with the dilatational velocity unaffected by the solenoidal field, is produced by small $M_{d}$. Accordingly, $M_{d} \rightarrow 0$ characterizes an acoustic regime for the dilatational part of the velocity field. The dilatational part of the energy remains bounded and close to its initial value, so that the increase of total energy is mainly due to its solenoidal part, which evolves independently of $M_{d}$. The relevance of this order-of-magnitude analysis has been verified using DNS for irrotationally distorted flows (Cambon et al. 1993); we find in what follows that for the case of pure shear it is also valid, but only for times short enough that the magnitude of the time-dependent wavevector $\boldsymbol{k}$ is adequately approximated by its initial value $k_{0}$. At later times, because of the coupling terms $m_{\alpha 3}$ (see (2.12)) and time-dependence of the coefficients that contain $k$, a more sophisticated analysis is needed to determine the large- $M_{d}$ behaviour. Such an analysis will be presented in $\S 5$.

\section{RDT code validation}

In this section analytic results are presented and used to verify the accuracy of the RDT code. This code (known as 'MITHRA') is an updated version of one used in previous linear-stability and RDT studies (cf. Cambon et al. 1985, 1994; Benoit 1992). Since the original has been thoroughly tested and validated for non-compressible cases, our focus here is upon flows for which the effects of compressibility are present.

We began by considering the case with no mean velocity gradients, which is characterized by pure-viscous decay for the solenoidal modes and oscillating solutions with possible damping and phase modification of coupled dilatation and pressure modes - as one might expect since the flow is composed of linear acoustic waves. As shown in Simone (1995), excellent agreement is found between the theoretical development of the solenoidal and dilatational modes and that predicted by the RDT code.

The complexity of the coupling terms in (2.12) increases as the deformation ranges from spherical compression to one-dimensional compression to pure shear (see figure 1); because analytic solutions exist for the first two, they provide ideal test cases for the RDT code, as we will now show. 
(a)

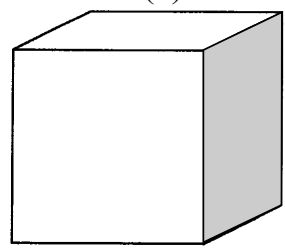

(b)

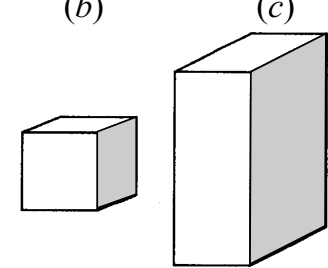

(d)

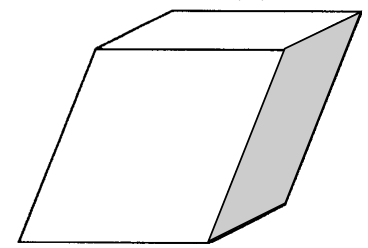

FIGURE 1. (a) Initial computational domain; (b) after an isotropic compression; (c) after a one-dimensional compression; $(d)$ after a pure shear.

\subsection{Spherical compression}

Mean spherical (isotropic) compression is defined by

$$
U_{i, j}=S \delta_{i j} ; \quad S(t)=\frac{S_{0}}{1+S_{0} t}=S_{0} J^{-1 / 3} ; \quad k_{i}=K_{i} J^{-1 / 3},
$$

where $\boldsymbol{K}=\left(K_{1}, K_{2}, K_{3}\right)$ is the initial wavenumber vector, $S_{0}$ is the rate of strain at $t=0$, and either the volumetric ratio $J(t)=\bar{\rho}(0) / \bar{\rho}(t)$ or the non-dimensional time $\left|S_{0}\right| t$ can be viewed as the time-advance parameter. We prefer the former, because it characterizes the mean spatial distortion at time $t$ as $\boldsymbol{F}$ does in (2.6), and yields simpler analytical RDT solutions. As for any irrotational mean flow, $m_{\alpha 3}=0$, so the solenoidal component of velocity is completely unaffected by the dilatational field. The evolution of the solenoidal kinetic energy is then easily found to be given by $q_{s}^{2}(t) / q_{s}^{2}(0)=J^{-2 / 3}(t)$ (where the zero subscript now indicates, as it will hereinafter, the initial value). In addition, $m_{3 \alpha}=0$, which implies that the equation for the dilatational field is also uninfluenced by the solenoidal velocity, and the right-hand side of (2.18) is zero. A closed-form solution for this case has been found and discussed by Blaisdell, Coleman \& Mansour (1996); its form is simplest when $\gamma$ is equal to $5 / 3$, so that $k^{2}$ and $a^{2}$ have the same $J^{-2 / 3}$ dependence, and simple solutions in terms of $\exp \left(\mathrm{i} a_{0} k(t) t\right)$ (where $k(t)=J^{-1 / 3}(t) K$, and $a_{0}$ is the initial mean sound speed) can be obtained for the modified dilatation and pressure modes $y=\widehat{\varphi}^{(3)} / J k$ and $z=\hat{p} / J \bar{\rho} a^{2}$. The resulting general solution for the transfer matrix $\boldsymbol{g}$, for the inviscid case, can then be written:

$$
\begin{array}{ll}
g_{\alpha \beta}=J^{2 / 3}(t) \delta_{\alpha \beta}, \quad \alpha, \beta=(1,2) \\
g_{33}=J^{2 / 3}(t) \cos \left(a_{0} k t\right) ; \quad g_{34}=-J^{2 / 3}(t) \sin \left(a_{0} k t\right) \\
g_{43}=J^{2 / 3}(t) \sin \left(a_{0} k t\right) ; \quad g_{44}=J^{2 / 3}(t) \cos \left(a_{0} k t\right) .
\end{array}
$$

As discussed in the previous section, all information about temporal evolution of the statistics is explicitly contained in these terms, with $J(t)$ accounting for the influence of the mean compression, and any oscillatory behaviour (which can be prevented by choosing acoustic-equilibrium initial conditions, such that $E^{(d)}=E^{(p)}$ at $t=0$ ) captured by the sine and cosine factors. The history of the dilatational kinetic energy can be derived from the initial dilatational field. Assuming that acoustic equilibrium holds for the initial conditions, one obtains the same variation found for the solenoidal energy, $q_{d}^{2}(t) / q_{d}^{2}(0)=J^{-2 / 3}(t)$. Having the initial data in acoustic equilibrium prevents temporal oscillations in the histories of kinetic and potential (pressure variance) energies. It also has the benefit of reducing the number of independent spectra needed to fully define the initial conditions (2.16). The resulting excellent agreement between analytical and numerical RDT results is shown in figures 2 and 3. Because the initial field is in acoustic equilibrium, the RDT solutions, the pressure-released 


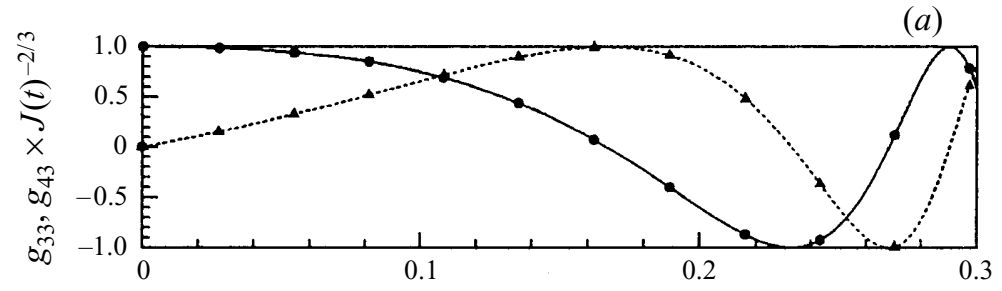

(b)

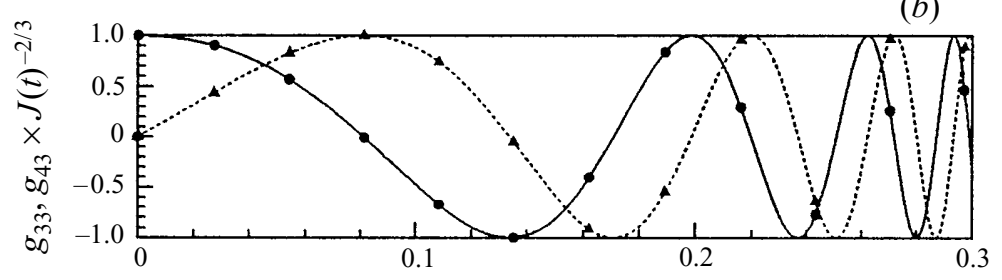

(c)

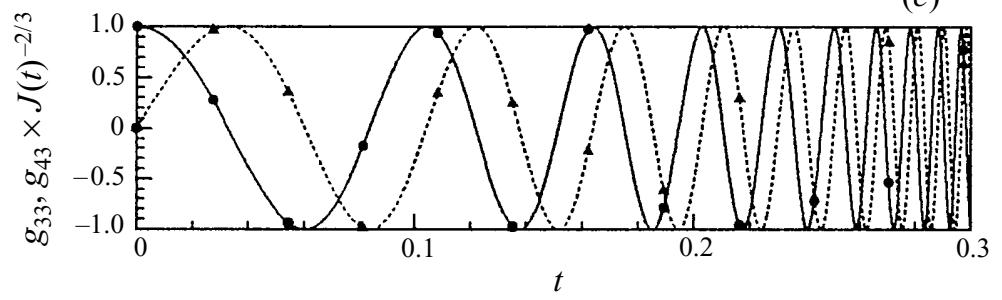

FIGURE 2. Histories, for three wavenumbers, of the $g_{33}$ and $g_{43}$ transfer matrix components for isotropic compression: -,$(3.2 a) ; \cdots \cdots,(3.2 b)$; symbols, numerical RDT results. $(a) k_{1} ;(b) k_{2}$; (c) $k_{3}$ with $k_{1}<k_{2}<k_{3}$.

approximation, and a WKB approximation (Durbin \& Zeman 1992) yield equivalent statistics; if the acoustic-equilibrium assumption is not made, however, differences occur. These differences, together with the role of $M_{d}$ for rapid spherical compressions, have been investigated in Blaisdell et al. (1996).

\subsection{One-dimensional axisymmetric compression}

The one-dimensional axisymmetric (axial) compression considered in this section is defined by

$$
U_{i, j}=S \delta_{i 1} \delta_{j 1} ; \quad S(t)=\frac{S_{0}}{1+S_{0} t}=S_{0} J^{-1} ; \quad k_{1}=K_{1} J^{-1} ; \quad k_{2}=K_{2} ; \quad k_{3}=K_{3},
$$

where the compression axis is in the $x_{1}$-direction, and $S_{0}$ again represents the initial compression rate. The solenoidal part of the velocity field is again independent of the dilatational component and closed-form solutions can be easily derived in terms of the volumetric ratio $J$. For the dilatational field, the solenoidal-dilatational coupling term $m_{3 \alpha}(\alpha=1,2)$ renders the solution more complex. In the previous section it was shown how the non-dimensional equation for $\widehat{\varphi}^{(3)}$ brings out the importance of the parameter $M_{d}$, which is equal to the ratio between the initial acoustic timescale and the mean distortion timescale; if $M_{d} \ll 1$, a pure-acoustic regime is obtained, independent of the evolution of the solenoidal mode, and the pressure and dilatational modes exchange energy at frequency $(a k)^{-1}$. The dilatational mode is exactly balanced by the pressure gradient given by the solution of the solenoidal Poisson equation in the pure-solenoidal case, and in this quasi-acoustic oscillatory (i.e. 'decoupled') regime 

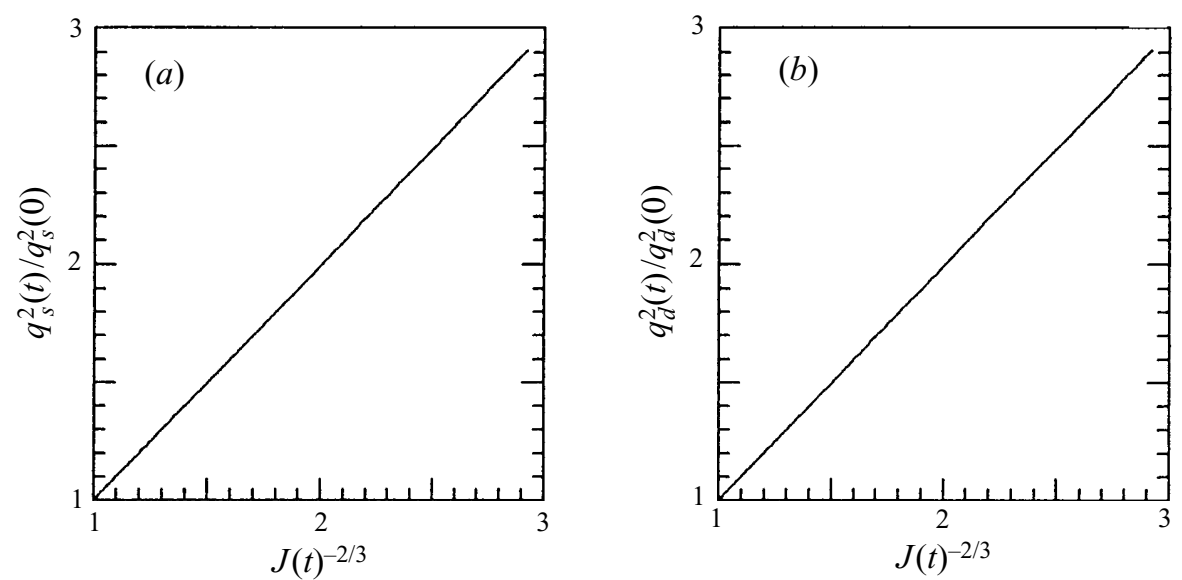

FIGURE 3. Solenoidal and dilatational turbulent kinetic energy histories predicted by RDT code for isotropic compression: $(a)$ solenoidal; $(b)$ dilatational for $\left(M_{t}, M_{d}\right)_{t=0}=(0.025,5)$.

$\begin{array}{ccrccc}\text { Case } & M_{t 0} & M_{d 0} & \left(\left|S_{0}\right| q^{2} / \epsilon\right)_{0} & (\operatorname{Re})_{0} & \left(q_{d}^{2} / q^{2}\right)_{0} \\ & & & & & \\ A_{1} & 0.025 & 5 & 194 & 358 & 0.06 \\ A_{2} & 0.11 & 87 & 800 & 184 & 0.18 \\ A_{3} & 0.29 & 29 & 100 & 500 & 0.09\end{array}$

TABLE 1. Initial physical parameters for axial-compression runs

(which is present at $M_{d} \ll 1$ for any irrotational strain) its energy is non-zero but bounded. If $M_{d} \gg 1$, the dilatational mode develops under the influence of the mean distortion without being constrained by the pressure, and its energy is added to that of the solenoidal mode, which is itself unaffected by $M_{d}$; this in turn leads to an increase, compared to the small- $M_{d}$ case, of the kinetic energy growth rate.

For the latter $M_{d} \gg 1$ 'pressure-released' limit (Jacquin et al. 1993), the maximum energy amplification occurs, such that

$$
\frac{q^{2}(t)}{q_{0}^{2}}=\frac{2+J^{-2}}{3}
$$

The other extreme, when $M_{d} \ll 1$ (referred to as the 'pseudo-acoustical' regime, with $q_{d}^{2} \ll q_{s}^{2}$ ), corresponds to a minimum energy amplification given by the pure-solenoidal RDT solution:

$$
\frac{q^{2}(t)}{q_{0}^{2}}=\frac{1}{2}\left(1+J^{-2} \frac{\tan ^{-1}\left(J^{-2}-1\right)^{1 / 2}}{\left(J^{-2}-1\right)^{1 / 2}}\right)
$$

(see Cambon et al. 1993, or Jacquin et al. 1993).

Tests of the RDT code have been performed by comparing (3.4) and (3.5) with the numerical results for axial compressions applied to various initial conditions; the initial velocity-correlation spectra used for the RDT runs were obtained from the DNS initial conditions described in Cambon et al. (1993). Table 1 lists the initial parameters for the three cases we have examined (corresponding respectively to runs A, B and C of Cambon et al. 1993). In figure 4, the total turbulent kinetic energy 


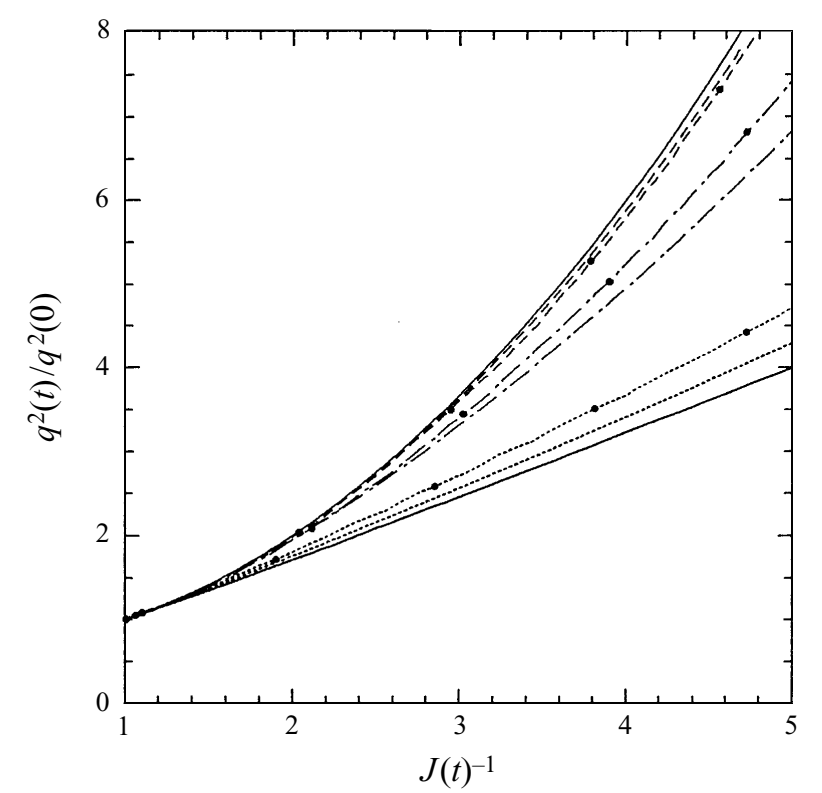

FiguRE 4. Turbulent kinetic energy histories for flow subjected to axial compression:- lower, (3.5); - upper, (3.4); DNS results: $\cdots \cdots$, case $A_{1} ;----$, case $A_{2} ;-\cdot-\cdot-$, case $A_{3}$; no symbols, numerical RDT results.

histories are plotted against the mean density ratio $J^{-1}$. Comparisons are made with the Cambon et al. (1993) DNS data and equations (3.4) and (3.5). The RDT code captures the same trends found in the analytic and DNS results: increased compressibility $\left(M_{d}\right)$ leads to an increase in the kinetic energy growth rate. Both solenoidal and dilatational contributions to the kinetic energy are shown in figure 5 and compared to DNS results. We see a strong amplification of the dilatational energy at the end of the compression, whereas the solenoidal field is unaffected by the dilatational field, as predicted by the RDT. This agreement of the theoretical, DNS and RDT results demonstrates the reliability of the code used to obtain the latter.

\section{Rapid-distortion theory and direct numerical simulation for pure-shear flow}

\subsection{RDT for pure-shear flow}

The pure-shear flow is defined by $U_{i, j}=S \delta_{i 1} \delta_{j 2}$ and $F_{i j}=\delta_{i j}+S t \delta_{i 1} \delta_{j 2}$. Without mean compression (i.e. $J(t)=1$ ) the appropriate time-advancing parameter is $S t, S$ being the constant shear rate $S=\mathrm{d} U_{1} / \mathrm{d} x_{2}$. The mean trajectories in physical and spectral space are

$$
x_{1}=X_{1}+S t X_{2}, \quad x_{2}=X_{2}, \quad x_{3}=X_{3}
$$

and

$$
k_{1}=K_{1}, \quad k_{2}=K_{2}-K_{1} S t, \quad k_{3}=K_{3} .
$$

General solutions for solenoidal RDT have been found for $\widehat{u}_{i}$ in a fixed frame of reference and extensively investigated (see e.g. Townsend 1976; Rogers 1991). Here solutions are obtained in terms of the solenoidal components $\varphi^{(\alpha)}(\alpha=1,2)$ and are most tractable when $\left(\boldsymbol{e}^{(1)}, \boldsymbol{e}^{(2)}\right)$ are chosen such that the polar axis $\boldsymbol{n}$ is in the cross- 


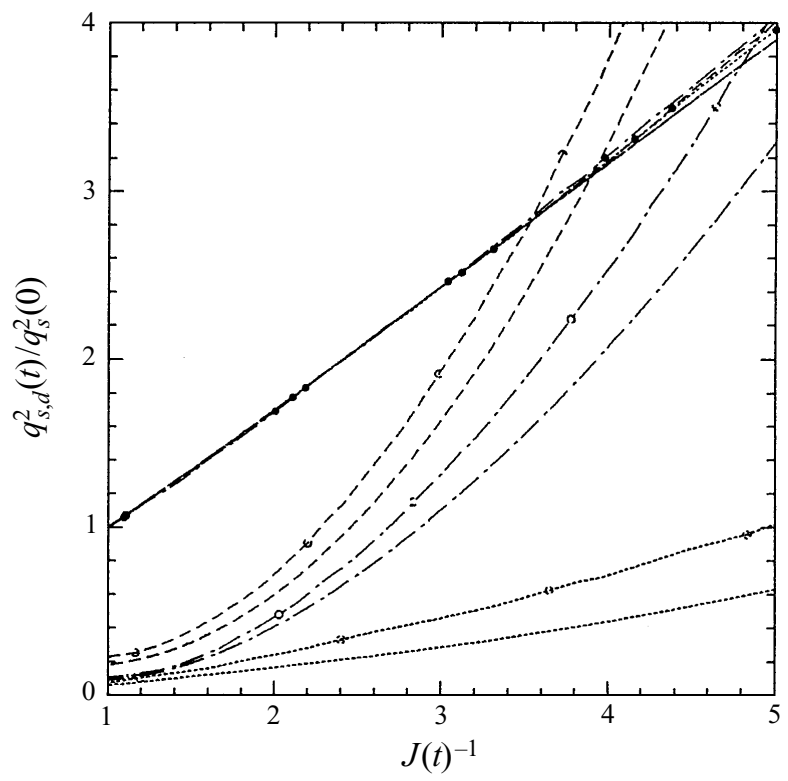

FIGURE 5. Solenoidal and dilatational turbulent kinetic energy histories for flow subjected to axial compression: $\bullet$, solenoidal; $\circ$, dilatational; DNS results: $\cdots$, case $A_{1} ;----$, case $A_{2} ;-\cdot-\cdot-$, case $A_{3}$; no symbols, numerical RDT results. (Note the collapse of the solenoidal TKE histories, as predicted by the RDT.)

gradient direction, with $n_{i}=\delta_{i 2}$, so that $e_{2}^{(1)}=0$. The corresponding physical meaning of $\varphi^{(\alpha)}$ will be discussed below. Substituting the mean velocity gradient for shear flow into (2.12), one obtains

$$
\begin{aligned}
\dot{\hat{\varphi}}^{(1)}+\frac{S K_{3}}{k} \widehat{\varphi}^{(2)} & =\frac{S k_{2} K_{3}}{K^{\prime}} y, \\
\left(k \dot{\hat{\varphi}}^{(2)}\right) & =-\frac{S K_{1}}{K^{\prime}} y, \\
\ddot{y}+a_{0}^{2} k^{2} y+2 \frac{S^{2} K_{1}^{2}}{k^{4}} y & =2 S \frac{\mathscr{D}}{\mathscr{D} t}\left(\frac{K_{1} K^{\prime}}{k^{4}}\right) k \widehat{\varphi}^{(2)}, \\
\dot{z} & =k^{2} y,
\end{aligned}
$$

where $y=\widehat{\varphi}^{(3)} / k, z=\widehat{\varphi}^{(4)} / a_{0}=\mathrm{i} \widehat{p} / \bar{\rho} a_{0}^{2}\left(\right.$ defined as in (2.17)) and $K^{\prime}=\left(K_{1}{ }^{2}+K_{3}{ }^{2}\right)^{1 / 2}$.

Unlike for a purely irrotational mean deformation, such as the two considered above, the non-zero coupling between the solenoidal and dilational velocity components causes the solenoidal field to no longer be uninfluenced by the dilatational field. The dilatational-solenoidal coupling is induced whenever the mean has a rotational component, since its general form $(2.13 b)$ is $m_{\alpha 3}=e_{i}^{(\alpha)}\left(U_{i, j}-U_{j, i}\right) e_{j}^{(3)}$; for the case of pure shear the coupling is expressed by the right-hand sides of $(4.3 a)$ and $(4.3 b)$. We shall see below that the coupling is not important at small $S t$, and depends only weakly upon $M_{d}$ for large $S t$, but otherwise its general behaviour is affected by $S t$ and initial $M_{d}$ in a manner that is difficult to predict without obtaining full RDT solutions.

The solenoidal limit is recovered for $y=0$, and the solenoidal RDT solution 
(Cambon 1982; Salhi \& Cambon 1996) is relatively simple:

$$
\begin{aligned}
& \widehat{\varphi}^{(1)}(\boldsymbol{k}, t)=\widehat{\varphi}^{1}(\boldsymbol{K}, 0)+\frac{K K_{3}}{K^{\prime} K_{1}}\left(\tan ^{-1}\left(\frac{k_{2}}{K^{\prime}}\right)-\tan ^{-1}\left(\frac{K_{2}}{K^{\prime}}\right)\right) \widehat{\varphi}^{(2)}(\boldsymbol{K}, 0), \\
& \widehat{\varphi}^{(2)}(\boldsymbol{k}, t)=\frac{K}{k} \widehat{\varphi}^{(2)}(\boldsymbol{K}, 0) .
\end{aligned}
$$

Using $e_{2}^{(1)}=0$ and $e_{2}^{(2)}=-K^{\prime} / k$ yields

$$
\widehat{\nabla^{2} u_{2}}=-k^{2} \widehat{u}_{2}=-k K^{\prime} \widehat{\varphi}^{(2)}-k k_{2} \widehat{\varphi}^{(3)}, \quad \widehat{\omega}_{2}=-\mathrm{i} K^{\prime} \widehat{\varphi}^{(1)},
$$

and shows that $k \widehat{\varphi}^{(2)}$ and $\widehat{\varphi}^{(1)}$ are linked to the variables $\nabla^{2} u_{2}$ and $\omega_{2}$, which are typically the bases for studying the stability of parallel shear flow with the OrrSommerfeld and Squire equations. In particular, the solenoidal $\left(y=0, \widehat{\varphi}^{(3)}=0\right)$ solution for the $\widehat{\varphi}^{(2)}$-equation is equivalent to conservation of $\nabla^{2} u_{2}$ along mean trajectories (as shown by $(4.5 a)$ and $(4.3 b)$ ).

The $M_{d} \gg 1$ pressure-released limit is found by neglecting the second term on the left-hand side in $(4.3 c)$, the equation governing $y$. This leads (for isotropic initial conditions) to quadratic amplification with respect to $S t$ of the turbulent kinetic energy,

$$
\frac{q^{2}(t)}{q_{0}^{2}}=1+\frac{(S t)^{2}}{3} .
$$

(When the initial conditions are not exactly isotropic, the pressure-released limit is given by $q^{2}(t) / q_{0}^{2}=1+\left(\overline{u_{2} u_{2}} / q^{2}\right)_{0}(S t)^{2}-2 S t\left(\overline{u_{1} u_{2}} / q^{2}\right)_{0}$.) A simple way to derive this $M_{d} \gg 1$ case is to ignore $p$ in $(2.1 a)$ such that

$$
\dot{u}_{1}+S u_{2}=0, \quad \dot{u}_{2}=\dot{u}_{3}=0,
$$

and use these expressions to obtain (4.6). Note that since $\mathscr{D} u_{2} / \mathscr{D} t=0$, the vertical velocity is advected in the pressure-released limit, whereas its Laplacian is advected in the solenoidal limit (for which $\mathscr{D}\left(\nabla^{2} u_{2}\right) / \mathscr{D} t=0$ ).

Unfortunately, the pressure-released limit yields the only easily derived analytical solutions for the Reynolds stress tensor. Hence numerical RDT solutions must be compared to DNS results for anything other than the $M_{d} \gg 1$ case. The system (4.3) will be re-examined, however, in $\S 5$.

\subsection{DNS for pure-shear flow}

The homogeneous shear flow data used for the ensuing analysis are obtained from DNS of the full non-isentropic compressible Navier-Stokes equations assuming spatial homogeneity. The simulations are generated by a version of the code developed by Spyropoulos \& Blaisdell (1996) (see also Blaisdell et al. 1991), which uses a pseudospectral spatial discretization with partial de-aliasing and a compact-storage thirdorder Runge-Kutta time advancement scheme. A time-dependent transformation is applied, yielding a coordinate system that moves with the mean shearing deformation (Rogallo 1981). This allows an expansion of the solution in Fourier series, and is equivalent to the above RDT procedure of using Lagrangian coordinates and the $F_{i j}$ mean-distortion tensor. Under the influence of the constant linear shear the shape of the domain in the streamwise/cross-gradient $(x, y)$-plane changes from a rectangle at time zero to a parallelogram at later times (cf. figure $1 d$ ). In the 


$\begin{array}{rrrrrr}\text { Case } & M_{t 0} & M_{d 0} & M_{g_{0}} & \left(S q^{2} / \epsilon\right)_{0} & R e_{t 0} \\ & & & & & \\ B_{0} & 0.25 & 2.7 & 0.4 & 10.7 & 296 \\ B_{1} & 0.25 & 4.0 & 0.6 & 16.0 & 296 \\ B_{2} & 0.25 & 8.3 & 1.3 & 33.1 & 296 \\ B_{3} & 0.25 & 12.0 & 1.9 & 48.0 & 296 \\ B_{4} & 0.25 & 16.5 & 2.6 & 66.2 & 296 \\ B_{5} & 0.25 & 24.0 & 3.8 & 96.1 & 296 \\ B_{6} & 0.25 & 32.0 & 5.1 & 128.1 & 296 \\ B_{7} & 0.25 & 42.7 & 6.8 & 170.8 & 296 \\ B_{8} & 0.25 & 53.4 & 8.5 & 213.5 & 296 \\ B_{9} & 0.25 & 66.7 & 10.6 & 266.9 & 296\end{array}$

TABLE 2. DNS initial parameters for pure-shear runs. Quantity $M_{d 0}$ based on large-eddy lengthscale $q^{3} / \epsilon ; M_{g_{0}}$ computed using cross-gradient integral lengthscale, as in Sarkar (1995). (Initial field obtained from unstrained precomputation.)

cross-gradient/spanwise $(y, z)$-plane, the domain always remains square. When the angle between the initially Cartesian $x$ - and $y$-axes decreases to $45^{\circ}$, the solution is projected onto an alternative domain of the same volume that is skewed 'backwards' so that the $x, y$ angle is $-45^{\circ}$; the simulation is then continued until the domain is again skewed to the point that the procedure must be repeated (Rogallo 1981). This 'remeshing', which is necessary to avoid grids with aspect ratios that approach infinity, produces a loss of energy at high wavenumbers and therefore leads to a discontinuity in temporal statistics (Blaisdell et al. 1991). However, as will be seen below, the practical importance of this uncertainty in the DNS results is limited, and does not adversely affect any of the conclusions of this study.

Intrinsic parameters that characterize the flow include the initial turbulent Mach number $M_{t 0}=q_{0} / a_{0}$ (recall that $\frac{1}{2} q_{0}^{2}$ is the initial turbulent kinetic energy and $a_{0}$ the initial mean speed of sound); the initial distortion Mach number $M_{d 0}=M_{t 0} S q_{0}^{2} / \epsilon_{0}$ ( $S$ is the constant mean shear and $\epsilon_{0}$ the initial total (solenoidal plus dilatational) rate of turbulent kinetic energy dissipation); and the initial turbulent Reynolds number $\left(R e_{t}\right)_{0}=q_{0}{ }^{4} / v \epsilon_{0}$. The ratio of the distortion Mach number to the turbulent Mach number $r=S q_{0}^{2} / \epsilon_{0}$ characterizes the rapidity of the shear. A series of ten simulations, denoted $B_{0}$ to $B_{9}$, are performed. The non-dimensional parameters $M_{t 0}, M_{d 0}$, and $R e_{t 0}$ defining the ten cases are given in table 2; also included are the initial nondimensional strain rate $\left(S q^{2} / \epsilon\right)_{0}$ and to help facilitate comparison to Sarkar's earlier DNS study, the equivalent values of the initial gradient Mach number $M_{g_{0}}$ he used to define his cases (using a transverse integral lengthscale; see below). The fluid is assumed to be an ideal gas with ratio of specific heats $\gamma=1.4$ and a temperaturedependent viscosity $\mu \propto T^{0.67}$. The Prandtl number is set to 0.7. Each simulation has the same initial $M_{t}$, equal to 0.25. Following Sarkar (1995), the initial distortion Mach number is progressively increased for cases $B_{0}$ to $B_{9}$ by modifying the shear rate $S$, all other non-dimensional parameters being unchanged. The initial distortion Mach number varies from 4 to 67 ; the initial shear rapidity ranges from $r=11$ to 267 . Each run uses a $96^{3}$ mesh overlaying the computational domain, with streamwise, cross-gradient and spanwise lengths in the ratio $2: 1: 1$.

Initial conditions are obtained from isotropic turbulence that has evolved from purely solenoidal velocity fields to ones that are to a good approximation in acoustic equilibrium. These initial fields are generated by running the code with no mean 
straining for about 2 units of 'eddy turn-over time' $q^{2} / \epsilon$ (measured in terms of quantities at the time the shear is applied), until they develop realistic triple-velocity correlations and dilatational energy for the given turbulent Mach number. This would be unnecessary if we were only interested in asymptotic behaviour (such as the $M_{d}$ dependence at large $S t$ examined by Sarkar 1995), since the later stages of sheared homogeneous compressible turbulence evolve to become independent of the initial data, allowing one to apply the shear directly to 'unphysical' initial conditions (Blaisdell et al. 1991; Sarkar 1995). But since here our interest is in both early and late times, the unstrained 'precomputations' are required. They begin from uniform density and pressure fields, and a random solenoidal velocity field, all of whose spectra are proportional to $E(k)=k^{4} \exp \left(-2 k^{2} / k_{p}^{2}\right)$; the peak wavenumber, $k_{p}$, locates the maximum initial value of the spectra, and is chosen such that $k_{p} L_{y} / 2 \pi=8$, where $L_{y}$ is the size of the domain in the cross-gradient (and spanwise) direction. Choosing this relatively small value of $k_{p}$ allows us to obtain Reynolds numbers larger than if $k_{p}$ had been larger (cf. Blaisdell et al. 1993; Sarkar 1995); however, because the streamwise lengthscales of the turbulence grow under the influence of the shear, this means that the periodic boundary conditions affect the solution sooner than they would for a larger- $k_{p}$ computation. Consequently, we have taken care to ensure that the DNS results presented below are independent of the domain size.

In order to explore the relevance of the RDT to Sarkar's (1995) DNS results, two of the simulations, cases $B_{1}$ and $B_{2}$, are respectively similar to his runs $A_{3}$ and $A_{4}$. The initial $M_{d}$ is thus chosen to be 4 for case $B_{1}$ and 8.3 for case $B_{2}$. Because our measure of the shear rapidity is based on the large-eddy lengthscale $\ell=q^{3} / \epsilon$, instead of the integral lengthscale of the velocity in the transverse shearing (cross-gradient) direction used by Sarkar to compute his gradient Mach number $M_{g}$, these values of $M_{d}$ closely correspond to the $M_{g}$ of 0.66 and 1.32 of his runs $A_{3}$ and $A_{4}$, respectively. (As we shall see, these two simulations by Sarkar can be considered as falling within the rapid-distortion regime, in the sense that they give results that are closely approximated by RDT solutions that use his case $A_{3}$ and $A_{4}$ parameters.) Although for the parallel $B_{1} / A_{3}$ and $B_{2} / A_{4}$ cases the effective initial distortion Mach numbers are approximately the same, the turbulent Mach numbers $M_{t}$ for Sarkar's and the present results are not. This is mainly a consequence of choosing to employ fully developed initial conditions; since a finite time is required for the unstrained precomputation to develop realistic turbulence, and during this time the turbulent Mach number rapidly decays, the initial turbulent Mach number used here, $M_{t 0}=0.25$, is smaller than the constant $M_{t 0}=0.4$ employed by Sarkar. However, when the distortion Mach numbers match, because the initial $M_{t}$ for the previous and present DNS are of the same order, so are the initial $M_{g} / M_{t}$ ratios. Because the peak in the initial spectra was chosen to be at a fairly low wavenumber, $k_{p}=16 \pi / L_{y}$, the turbulent Reynolds number $\operatorname{Re}_{t} \approx 300$ of the initial field generated by the precomputation is significantly larger than the initial $R e_{t} \approx 200$ used by Sarkar. Spectra were examined to verify that at this Reynolds number the $96^{3}$-grid is sufficient to accurately resolve both the initial and sheared turbulent fields. It was mentioned earlier that during the computations the integral lengthscales grow so that eventually the large eddies fill the computational domain and the simulations become invalid. The density integral-lengthscale is a good measure of whether or not this has happened (Blaisdell et al. 1991). Accordingly, the two-point correlation of the density field was monitored to verify that the solutions are not adversely influenced by the periodic boundary conditions before the time at which each simulation was stopped, which varied between $S t=10$ and 15 . 


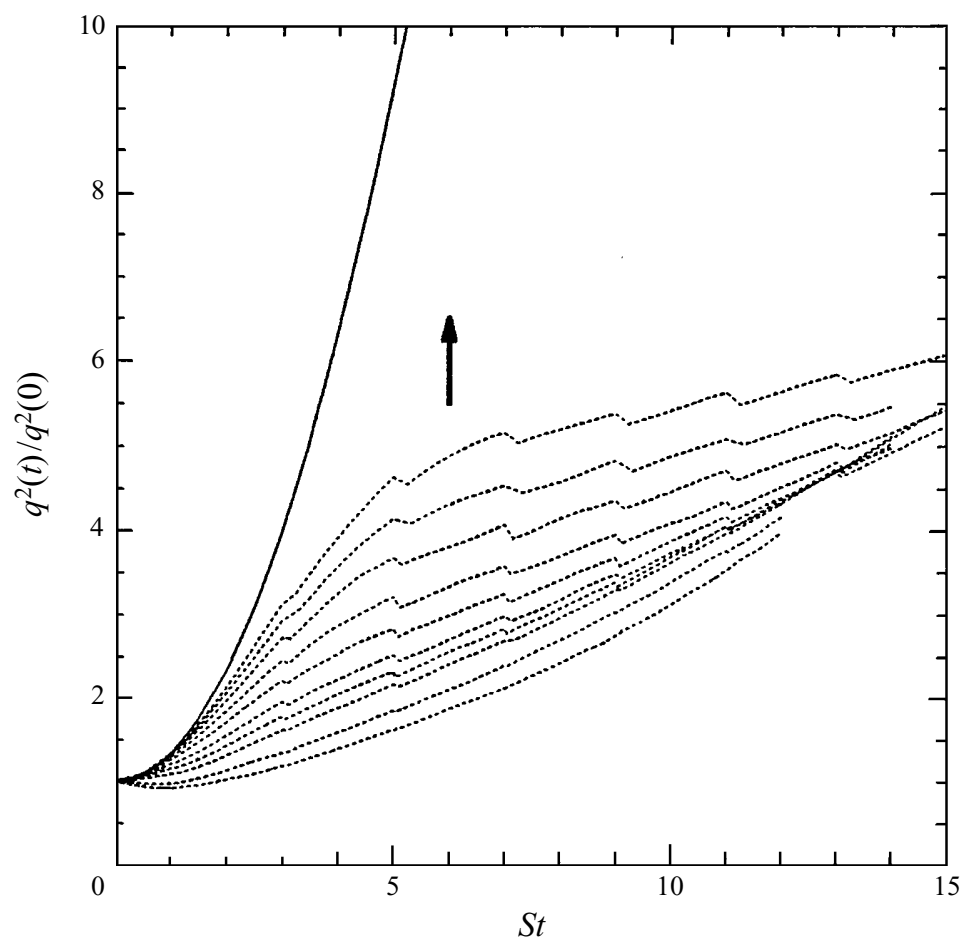

FIGURE 6. Turbulent kinetic energy histories for pure-shear flow: —_, (4.6); $\cdots$, DNS with initial $M_{d}$ ranging from 4 (lower) to 67 (upper). Arrow shows trend with increasing $M_{d}$.

\subsection{Numerical RDT and DNS results}

Histories of the total turbulent kinetic energy from the DNS are presented in figure 6, plotted against the non-dimensional shear rate St. (The discontinuity at odd values of $S t$ is caused by the remeshing procedure, explained above.) As predicted by the RDT analysis in $\S 4.1$, we find that all the curves are bounded by the pressure-released $\left(M_{d} \gg 1\right)$ limit represented by the solid curve. The rate of energy amplification monotonically increases with initial $M_{d}$, at low $S t$, and becomes linear at large $S t$. The linear growth rate is much smaller, however (by about a factor of three for the lowest- $M_{d}$ DNS run at $S t \approx 12$, for example) than the large-St RDT prediction for the incompressible case, $\partial\left(q^{2} / q^{2}(0)\right) / \partial(S t) \sim 2 \ln 2$ (Rogers 1991). In fact, both the asymptotic incompressible RDT solution and that at general $S t$ consistently overpredict the magnitude of $q^{2}(t) / q^{2}(0)$ observed in the compressible DNS. However, we shall subsequently see that the history of the TKE production rate given by the solenoidal RDT analysis is relevant to the fully compressible flow.

The development of the temporal growth rate of the turbulent kinetic energy is shown in figures 7 $(a)$ and 7(b) (only DNS results not directly affected by the remeshing discontinuity are included), and will be discussed further below. (The oscillations in the DNS results are due to the statistical uncertainty associated with the limited sample provided by a domain of finite size, while the slight 'waviness' at large times in the RDT histories is the result of the wavenumber discretization (equidistant in $\log k$, see Cambon 1982; Benoit 1992) needed to solve (2.12), and also the difficulty in resolving at large wavenumber the high frequencies $a k$ introduced at large $S t$.) The 

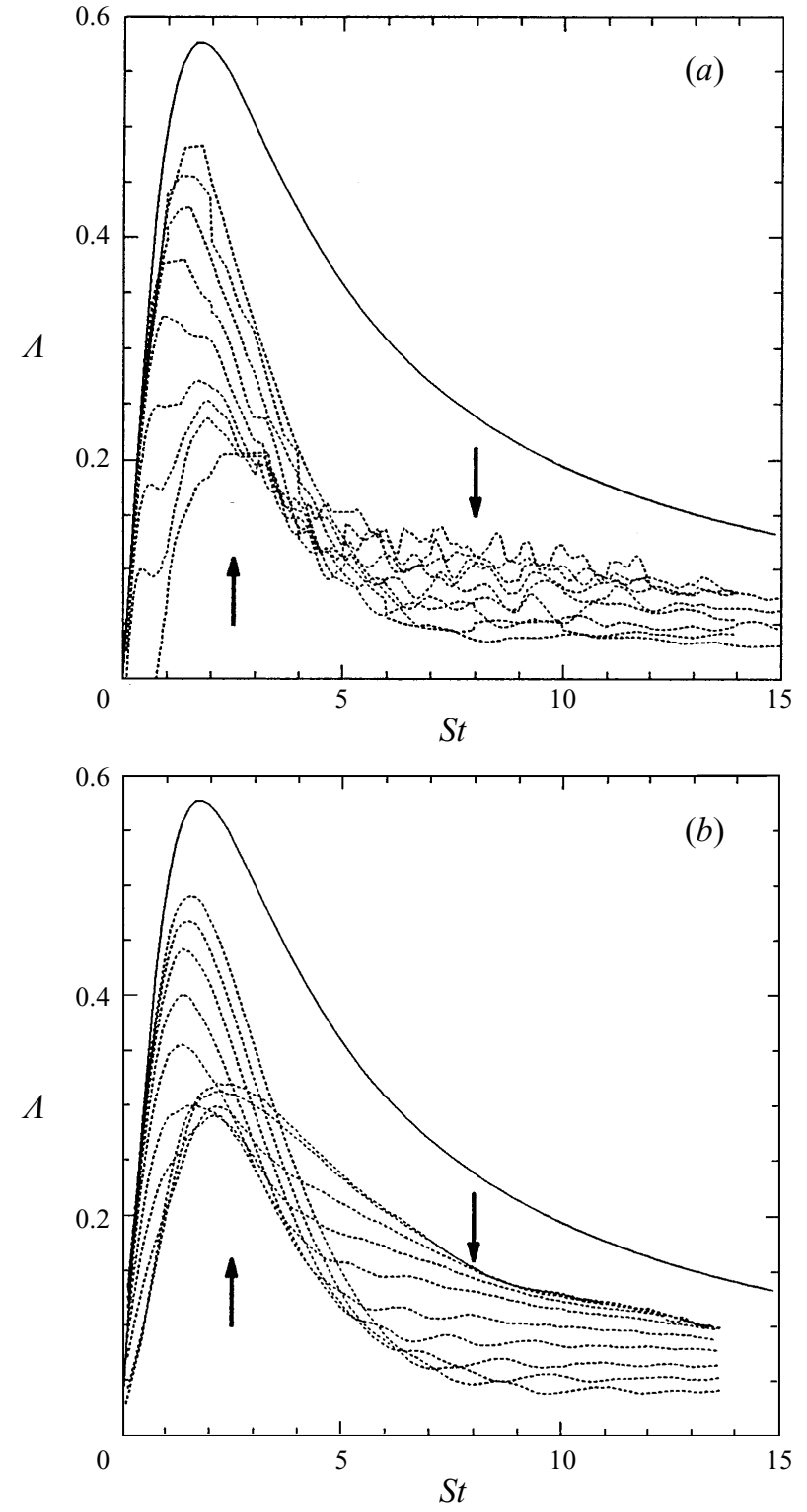

FIGURE 7. Histories of the temporal energy growth rate for pure-shear flow:,$-(4.9) ;(a) \cdots \cdots$, DNS; $(b) \cdots$, RDT. Initial $M_{d}$ ranges from 4 to 67 for both DNS and RDT; arrows show trend with increasing $M_{d}$.

growth rate is defined by the non-dimensional parameter (as in Sarkar's paper)

$$
\Lambda=\frac{1}{S \mathscr{K}} \frac{\mathrm{d} \mathscr{K}}{\mathrm{d} t},
$$

where $\mathscr{K}=\frac{1}{2} q^{2}$. Figure 7 indicates that for $S t<4$ the growth rate increases with initial distortion Mach number $M_{d 0}$ and tends toward the pressure-released limit 


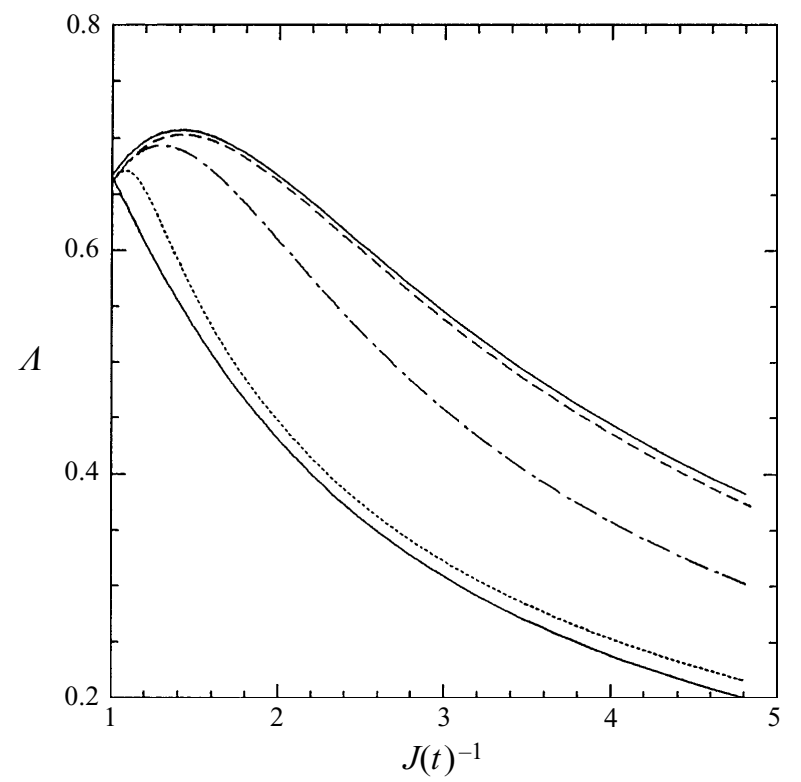

FIGURE 8. RDT histories of the temporal energy growth rate for one-dimensional axially compressed flow: - analytic RDT limits: lower, pure-solenoidal regime; upper, pressure-released regime; $\cdots$, case $A_{1}, ;----$, case $A_{2} ;-\cdot-\cdot \cdot$, case $A_{3}$.

(solid curve) given by

$$
\Lambda^{p r}=\frac{2 S t}{3+(S t)^{2}},
$$

which represents the maximum growth rate, found for initial $M_{d 0} \gg 1$. However, the trend is reversed at larger $S t: \Lambda$ decreases with increasing $M_{d 0}$. It is noteworthy that all the curves exhibit this transition from increasing to decreasing growth rate with $M_{d}$ near $S t \approx 4$ or 5 . This 'crossover' feature can only exist when the mean velocity field is rotational; to illustrate, RDT histories of the temporal energy growth rate for the one-dimensional axisymmetric compression discussed above (cases $A_{1}$ to $A_{3}$ ) are presented in figure 8 . Here $\Lambda$ is observed to scale monotonically with $M_{d 0}$, which varies from 0 for the lower solid curve, to 5 for the dotted, 29 for the chain-dotted, 87 for the dashed, and infinity for the upper solid curve (the two theoretical curves - the $M_{d}=0$ solenoidal and $M_{d} \rightarrow \infty$ pressure-released limits - being obtained by taking the time derivative of (3.5) and (3.4), respectively). The implication is that the crossover is due to the coupling of the solenoidal and dilatational velocity fields mediated by $m_{\alpha 3}$, which is equal to zero if the mean flow is irrotational.

What is thought to be one of the most noteworthy results of this study is found in figure 7, which shows that the general $M_{d}$ dependence observed in the homogeneousshear DNS is also present in the RDT. Although there are some quantitative differences between the RDT and DNS data (the DNS growth rates are consistently smaller than those predicted by the RDT, and consequently the asymptotic values of $\Lambda$ at large $S t$ are not the same), in general the agreement is quite striking, even in the approximate location of the $S t \approx 4$ crossover transition.

Continuing our examination of the DNS and RDT results and their implications, we next use (1.4) to derive the exact equation for the evolution of $\Lambda$ in homogeneous 

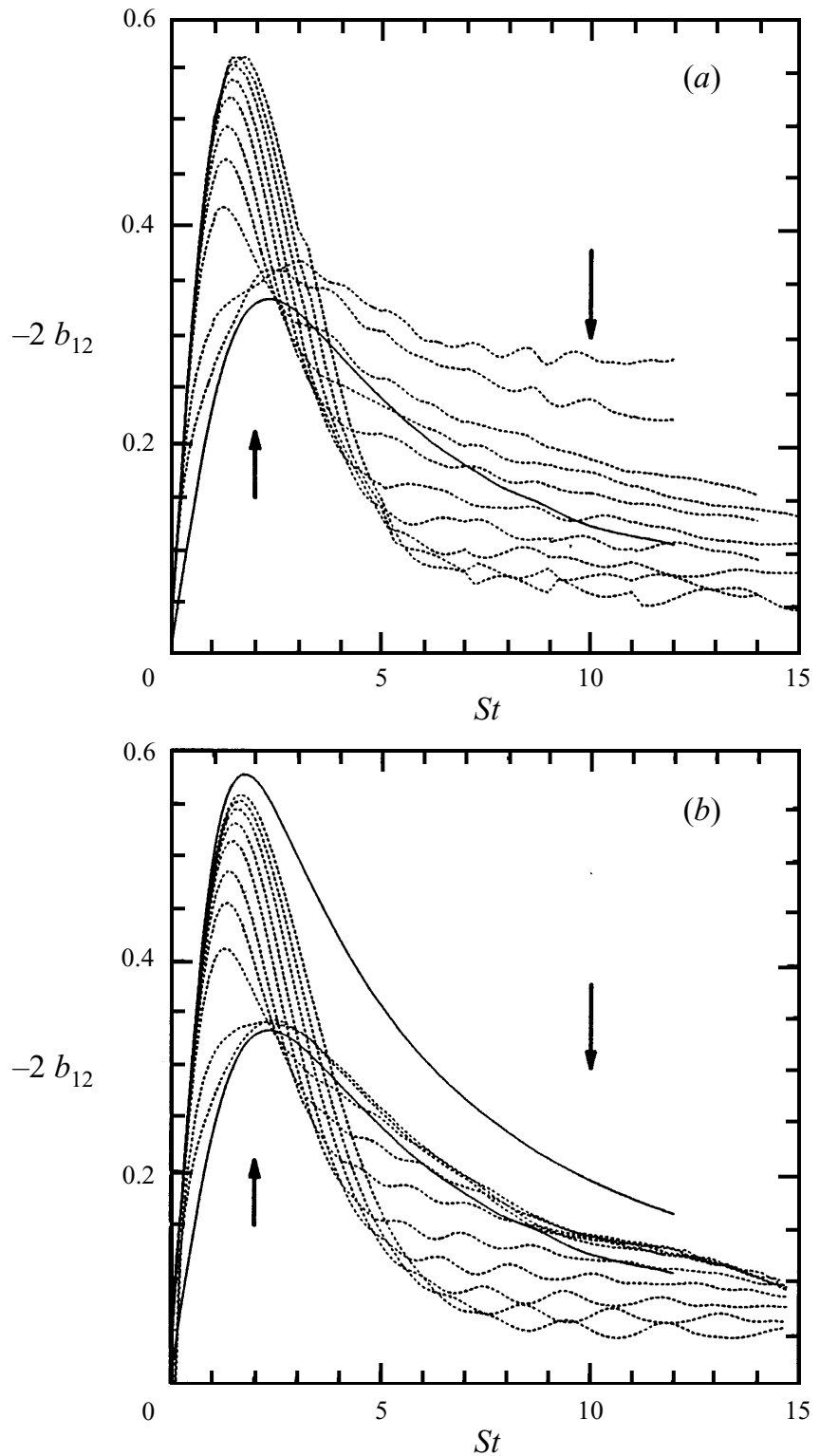

FiguRE 9. Histories of the non-dimensional production term $-2 b_{12}$ for pure-shear flow: $(a) \cdots \cdots$, DNS; $(b) \cdots$, RDT; — lower, incompressible run performed using the incompressible-flow version of MITHRA; - upper, pressure-released limit. Initial $M_{d}$ ranges from 4 to 67 for both DNS and RDT; arrows show trend with increasing $M_{d}$.

shear flow:

$$
\begin{aligned}
\Lambda & =-2 b_{12}-\frac{\epsilon_{s}+\epsilon_{d}-\Pi_{d}}{S \mathscr{K}} \\
& =-2 b_{12}\left(1-\frac{\epsilon_{s}+\epsilon_{d}-\Pi_{d}}{\mathscr{P}}\right)=-2 b_{12}\left(1-\chi_{\epsilon}\right),
\end{aligned}
$$

where $b_{12}=\overline{u_{1} u_{2}} / 2 \mathscr{K}=-\mathscr{P} / 2 S \mathscr{K}$ is the relevant component of the Reynolds 


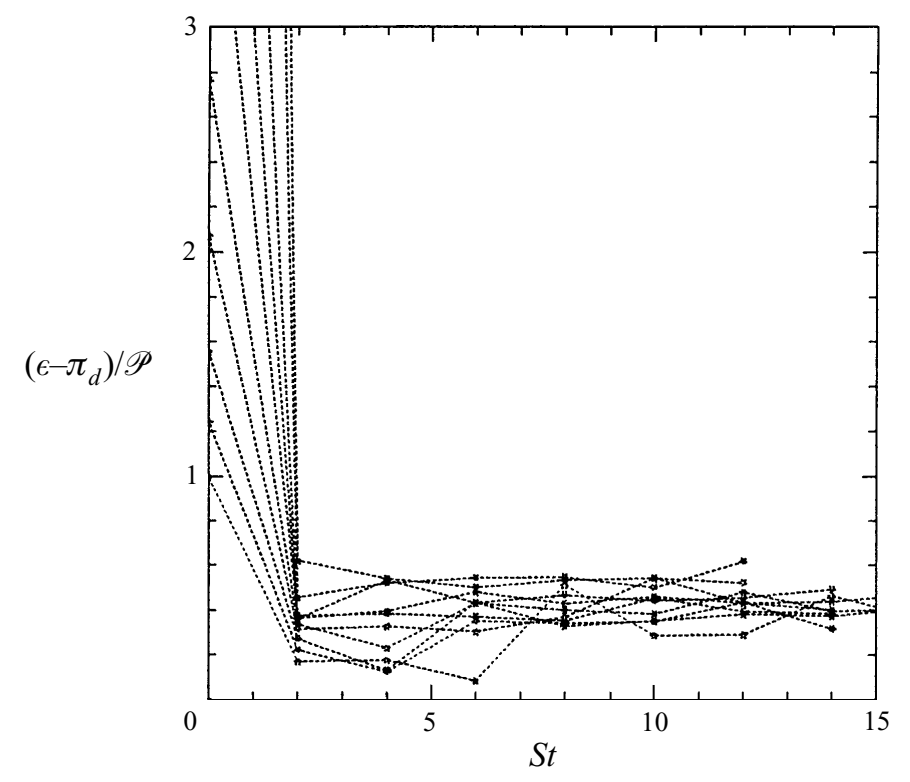

FIGURE 10. DNS histories of the sum of pressure-dilatation terms and rate of kinetic energy dissipation, scaled by the production term, for pure-shear flow. Lines included only as an aid to viewing, to reveal trends with $M_{d}$.

stress anisotropy tensor, which represents the non-dimensional production term; $\chi_{\epsilon}=\left(\epsilon_{s}+\epsilon_{d}-\Pi_{d}\right) / \mathscr{P}$ is the sum of the total (solenoidal plus dilatational) dissipation rate and the pressure-dilatation correlation, scaled by the production term. Tavoularis $\&$ Corrsin (1981) have shown that in incompressible homogeneous shear flow $b_{12}$ and $\epsilon_{s} / S \mathscr{K}$ tend toward equilibrium values that are independent of initial conditions. As first found by Sarkar (1995), and demonstrated in figures 7(a) and 7(b), $\Lambda$ also approaches a constant asymptotic value $\Lambda_{\infty}$ in the compressible case, with $\Lambda_{\infty}$ depending on $M_{d}$. According to (4.10), the change in $\Lambda_{\infty}$ with compressibility (i.e. $M_{d}$ ) can be due either to a change in $b_{12}$ - and thus in the production - or to a change in the solenoidal dissipation and dilatational effects represented by $\chi_{\epsilon}$. DNS histories of $-2 b_{12}$ are plotted as the broken curves in figure $9(a)$, and compared to the RDT histories (figure $9 b$ ). The evolution of the corresponding term for incompressible homogeneous shear flow, which was generated using the incompressible-flow version of the RDT code (Cambon et al. 1985), and the pressure-release limit, are also shown, by the solid curves. The magnitude of the non-dimensional production is found to increase with increasing $M_{d 0}$, for all the cases considerated for $S t<4$ or 5 , while at large $S t$, the turbulent kinetic energy production by the mean shear is systematically reduced as $M_{d 0}$ increases. As was the case for the $\Lambda$-histories, the $S t \approx 4$ transition found in the DNS is also predicted quite well by RDT, although the underestimation by the RDT of the stabilizing effect of compressibility via reduced production becomes more pronounced with increasing St. The lack of complete quantitative agreement notwithstanding, the general correspondence between the DNS and RDT histories of $b_{12}$ is perhaps the most significant (and surprising) result of this study.

The other mechanism present in (4.10) is investigated in figure 10, where DNS histories of $\chi_{\epsilon}$ are shown. After an initial transient, $\chi_{\epsilon}$ tends toward a constant value, independent of the initial distortion Mach number. This implies that the reduction of amplification of energy growth rate with increasing compressibility (with respect to 

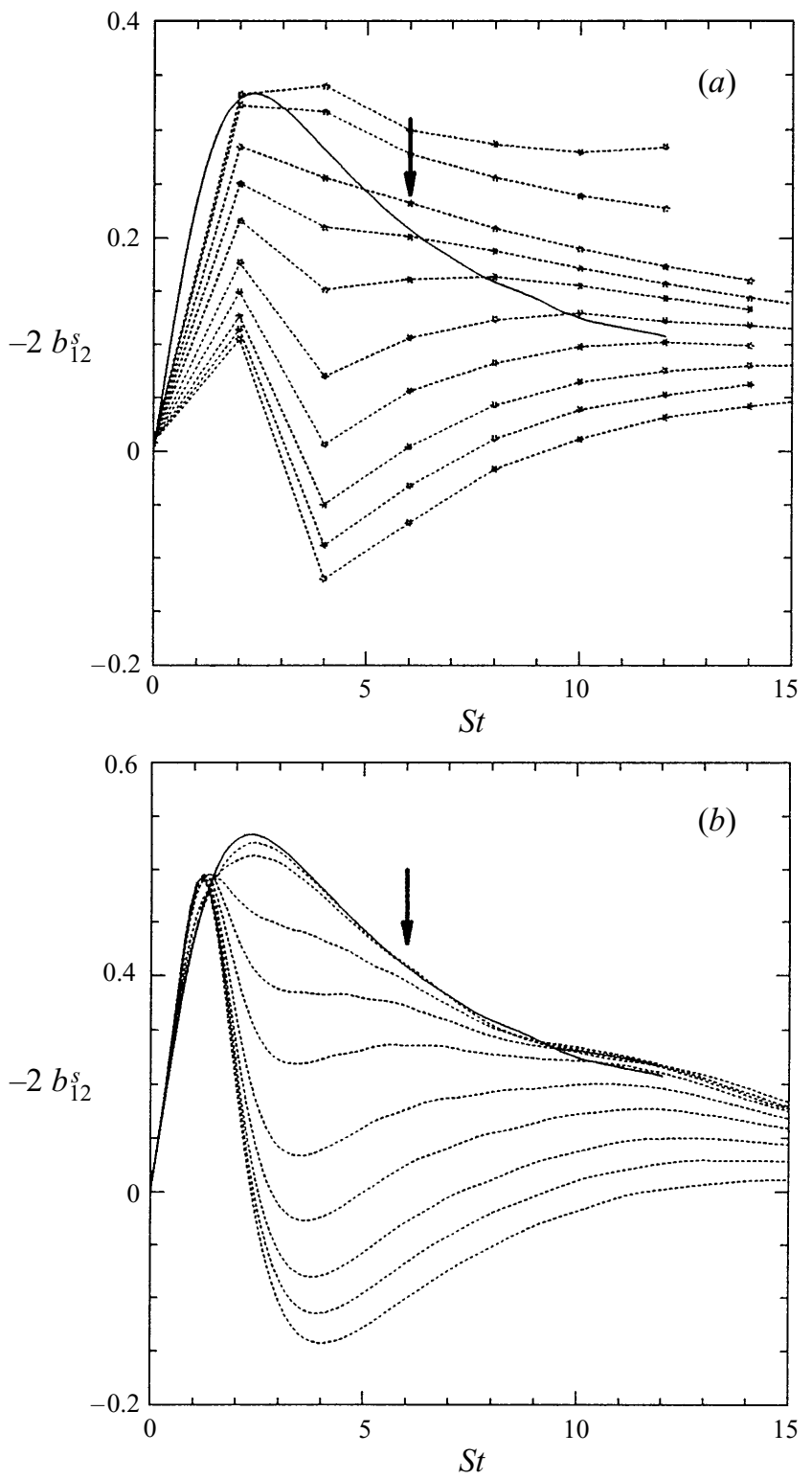

FIGURE 11. Histories of solenoidal $b_{12}$-component anisotropy tensor for pure-shear flow: incompressible run performed using the incompressible-flow version of MITHRA; $(a) \cdots \cdots$, DNS; (b) $\cdots$, , RDT. Initial $M_{d}$ ranges from 4 to 67 for both DNS and RDT; arrows show trend with increasing $M_{d}$. Lines in (a) included only as an aid to viewing, to reveal trends with $M_{d}$.

the incompressible case) is mainly due to less efficient production, through reduction of the anisotropy of the Reynolds stress tensor. In other words, changes in the explicit compressibility terms - those that appear as sources or sinks in the turbulent kinetic energy equation - are not as important as compressibility-induced changes to the the structure of the flow. As first revealed by Sarkar's earlier DNS study, it is the kinetic energy production that is primarily affected by compressibility.

In an attempt to separate the effect of compressibility on the solenoidal and dilata- 
tional contributions to the turbulent kinetic energy, the Helmholtz decomposition of the fluctuating velocity field was used for the pure shear as well as the one-dimensional compression flow; no evidence of scaling with $M_{d 0}$ was found. However, decomposition of the $b_{12}$ component of the anisotropy tensor into solenoidal and dilatational components defined by

$$
b_{i j}{ }^{(s)}(t)=\frac{R_{i j}{ }^{(s)}(t)}{q_{s}^{2}(t)}-\frac{\delta_{i j}}{3}, \quad b_{i j}{ }^{(d)}(t)=\frac{R_{i j}{ }^{(d)}(t)}{q_{d}^{2}(t)}-\frac{\delta_{i j}}{3}
$$

(where $R_{i j}^{(s)}=\overline{u_{i}^{s} u_{j}^{s}}$ and $R_{i j}^{(d)}=\overline{u_{i}^{d} u_{j}^{d}}$ ) is more fruitful. Histories of these terms are shown in figures 11 and 12 , respectively. We see that the solenoidal contribution is dramatically decreased over the entire range of $S t$ whereas the dilatational one is essentially unaffected by compressibility. The structure alteration mentioned above is therefore due almost entirely to changes in the solenoidal field. For all the cases considered both contributions approach constant equilibrium values at large $S t$. The RDT predicts that the dilatational component is essentially independent of $M_{d}$ for all values of $S t$, while the solenoidal component exhibits a pronounced $M_{d}$ dependence over the entire range of times shown, with the strong suggestion of collapse to a unique value (independent of $M_{d}$ ), as $S t \rightarrow \infty$. The DNS results, on the other hand, while showing the same general trends as the RDT histories do, tend to be more sensitive to $M_{d}$, with the large-St limits of both $b_{12}^{(s)}$ and $b_{12}^{(d)}$ depending upon the initial distortion Mach number. This difference between the RDT and DNS results is probably due to the nonlinear and dissipative terms that are present in the DNS; the importance of these terms will be discussed in the next section.

\section{Discussion and conclusions}

\subsection{General comments}

The objective of this work has been to develop a general RDT code capable of solving linearized equations valid for compressible homogeneous turbulence for a large range of the two compressibility parameters important for turbulence subjected to mean shear, $M_{t}$ and $M_{d}$. Two types of mean uniform velocity gradients (spherical compression and one-dimensional axisymmetric compression) have been examined in order to validate the numerical code; results of the RDT code have been compared with analytical solutions and DNS data at high compression speed, and have demonstrated good agreement. The pure plane-shear flow was then investigated and RDT and DNS results have been presented and discussed in the light of recent simulations performed by Sarkar (1995). As in his earlier study, we find that changes in the 'structure' of the turbulence (i.e. altered production of turbulent kinetic energy) are much more significant than changes associated with the new (compressibility-induced) source terms in the turbulent kinetic energy equation.

When viewed separately, the primary value of the RDT and DNS results presented here is solely to reconfirm (and extend the range of validity for) many of Sarkar's earlier findings regarding the structure-altering role of compressibility in homogeneous sheared turbulence. However, when they are viewed together, their broad general agreement reveals the extent to which the structural alterations are governed by linear processes. Although the agreement is not exact (for example, in the $S t \rightarrow \infty$ asymptotic limits predicted for $\Lambda$ and $b_{12}$ ), many of the RDT and DNS results are surprisingly close to each other. In particular, we note that at early times the RDT and DNS histories are very similar, and especially that the RDT correctly locates 

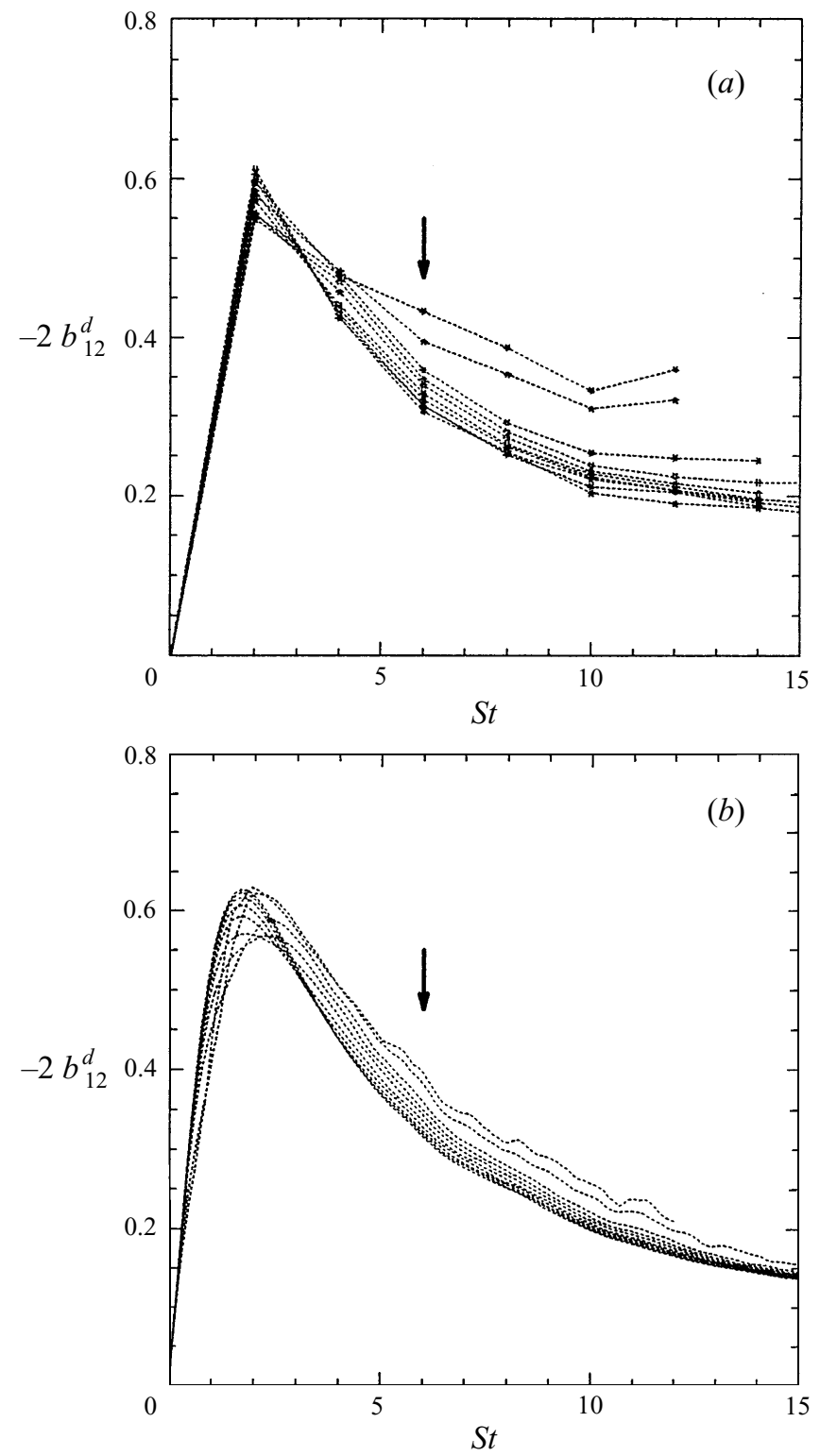

FIGURE 12. Histories of dilatational $b_{12}$-component anisotropy tensor for pure-shear flow: legend as in figure 11.

the $S t \approx 4$ 'crossover' transition that indicates the time at which the influence of increased compressibility (i.e. $M_{d}$ ) changes from destabilizing to stabilizing. Because of the similarity of the RDT and DNS solutions, we conclude that much of the effect of compressibility (at least that which influences the TKE production and shear-stress anisotropy $b_{12}$ ), which was heretofore commonly thought to be due to nonlinear phenomena, can be explained in terms of linear rapid-distortion theory. This is a central conclusion of this paper. It is important to realize that the magnitudes of the compressibility parameters $\left(M_{t}\right.$ and $\left.M_{d}\right)$ considered in this study are not unrepresentative of those found in engineering flows of practical interest; for example, 
based on the analysis of Sarkar (1995), we estimate that the distortion Mach numbers employed for cases $B_{1}$ and $B_{2}$ (cf. table 2) correspond to convective Mach numbers found in a compressible mixing layer of less than one (see his figure 14). The present study therefore appears to be much more relevant than one might expect of an analysis based on rapid-distortion assumptions. Given this relevance, we now turn our attention to its broader implications.

\subsection{The 'stabilizing' effect of compressibility revisited}

A comparison of the development of the growth-rate parameter $\Lambda$ between RDT results for axisymmetric compression (figure 8) and pure shear (figure $7 a, b$ ) illustrates that increases in the distortion Mach number can cause the turbulence to become either more or less energetic, depending upon both the type of mean deformation and the time at which the flow is examined. In this section, we attempt to clarify the reasons for, and the conditions that produce, both the stabilizing and destabilizing influence of compressibility. We begin by considering deformations for which increasing $M_{d}$ always causes the flow to become more energetic.

\subsubsection{Irrotational distortions}

Intrinsic compressibility is destabilizing - in the sense that it leads to generation of greater turbulent kinetic energy than when the velocity fluctuations are purely solenoidal - for all rapid irrotational mean distortions, throughout the history of the flow; because the solenoidal field is unaffected by an irrotational deformation, the sum of the solenoidal and dilatational kinetic energy will always be greater than that found for the purely solenoidal case. This can be understood, without having to utilize a Fourier representation, from the general solution of the linearized equations,

$$
\begin{aligned}
u_{i}(\boldsymbol{x}, t) & =\underbrace{F_{j i}^{-1}(\boldsymbol{X}, t, 0) u_{j}(\boldsymbol{X}, 0)}_{V_{i}=V_{i}^{(s)}+V_{i}^{(d)}}+\frac{\partial \phi}{\partial x_{i}}, \\
\dot{\phi} & =p / \bar{\rho},
\end{aligned}
$$

which is valid even for inhomogeneous flows. Equation (5.1) was extensively used by Goldstein (1978) and Durbin \& Zeman (1992), among others (the present notation follows that of Cambon 1982 and Cambon et al. 1985, 1993). The first term on the right-hand side of (5.1a) can be split into a solenoidal and a dilatational part (via the Helmholtz decomposition), such that $F_{j i}^{-1}\left(u_{j}\right)_{0}=V_{i}=V_{i}^{(s)}+V_{i}^{(d)}$; the dilatational part is present for any anisotropic mean strain even if the initial velocity field is solenoidal. Accordingly, the other term in $(5.1 a)$, which involves the pressure fluctuation, exactly balances the dilatational part $V_{i}^{(d)}$ in the solenoidal limit $M_{d} \rightarrow 0$, and is zero in the pressure-released regime $\left(M_{d} \rightarrow \infty\right)$. In other words, $V_{i}$ is the pressure-released RDT solution, and $V_{i}^{(s)}$ denotes the solenoidal RDT solution:

$$
u_{i}^{s}=V_{i}^{(s)}, \quad u_{i}^{d}=V_{i}^{(d)}+\phi_{, i} .
$$

The transition with increasing $M_{d}$ from the solenoidal to the pressure-released solution accompanies the progressive emergence of $u_{i}^{d}$, as $\boldsymbol{V}^{(d)}$ is less and less balanced by $\phi_{,}$, at fixed (since it is unaffected by compressibility) $u_{i}^{s}$. This suggests the simple parameterization:

$$
u_{i}=V_{i}+f\left(M_{d}\right)\left(V_{i}^{(s)}-V_{i}\right),
$$

where $f\left(M_{d}\right)$ is a monotonic function that varies from 1 to 0 as $M_{d}$ varies from 0 (representing the solenoidal solution) to infinity (yielding the pressure-released 
solution), with $V_{i}=F_{j i}^{-1}\left(u_{j}\right)_{0}$ and its solenoidal projection $V_{i}^{(s)}$ being determined by the Lagrangian distortion tensor and the initial conditions only. (Such a weightingfunction parameterization was suggested in a modified model for pressure-strain correlations by Cambon et al. 1993.) This simple analysis is sufficient to explain the main compressibility effects in RDT for irrotational mean flows. Further analysis, however, requires the use of Fourier space in order to decompose $V_{i}$ into $V_{i}^{(s)}+V_{i}^{(d)}$ in a tractable form, and especially to study the coupled system of equations governing $u_{i}^{d}$ and $p,(2.18)$ and (2.19).

\subsubsection{Vortical distortions: the pure shear}

The above simple analysis is no longer possible if the mean distortion has a vortical part. The Weber-Goldstein equation (5.1) is no longer valid, and the complete system (2.12) must be considered. However, the pure-shear RDT and DNS results shown above nevertheless exhibit the destabilizing trend predicted by (5.3), but only at small $S t$. At later times, for $S t$ greater than about 4, behaviour opposite to that implied by the analysis in the previous section is found (figure 7). The reason for this transition of the influence of compressibility from being destabilizing to being stabilizing will now be sought.

The essential difficulty in trying to analytically solve the linear system of RDT equations (4.3) for pure shear comes from the time-dependent character of the coefficients that contain $\boldsymbol{k}(t)$ (see (4.2)). Only when $k_{1}=0$ does this time-dependence disappear, so that (4.3) is drastically simplified, yielding an oscillatory solution for $y$ (as in the acoustic unsheared case), and the pure-solenoidal solution for $\widehat{\varphi}^{(2)}$. For $k_{1} \neq 0$ we focus on the restricted system of equations for $\widehat{\varphi}^{(i)}$, with $i=(2,3,4)$ only:

$$
\dot{\xi}=S K_{1} k^{2} y, \quad \dot{y}=-2 S \frac{K_{1}}{k^{4}} \xi-a_{0}^{2} z, \quad \dot{z}=k^{2} y,
$$

where $y=\widehat{\varphi}^{(3)} / k$ and $z=\widehat{\varphi}^{(4)} / a_{0}$ are defined as in (4.3), and $\xi=-k K^{\prime} \widehat{\varphi}^{(2)}$ is the Fourier transform of the solenoidal part of $\nabla^{2} u_{2}$, as shown in (4.5a). Only the vertical (i.e. cross-gradient) velocity component (through $\xi$ and $y$ ) and the pressure (through $z$ ) are present in the above, and the time-dependence of the coefficient is solely through $k^{2}(t)$. Because the time-dependent parts of the right-hand side of both $(5.4 a)$ and $(5.4 c)$ are in $k^{2} y$, the new variable

$$
Z=z-\frac{\xi}{S K_{1}}
$$

is time-invariant $(\dot{Z}=0)$, and can replace $z$ in the set of variables. Thus, in terms of $Z, \xi$ and $y$, the previous system can be written

$$
\dot{Z}=0, \quad \dot{\xi}=S K_{1} k^{2} y, \quad \dot{y}=-\left(2 S \frac{K_{1}}{k^{4}}+\frac{a_{0}^{2}}{S K_{1}}\right) \xi-a_{0}^{2} Z .
$$

The latter equation is the key to understanding the time-dependence of (linear) compressibility effects in turbulent shear flow; this is done by comparing the magnitude of the two terms in the $\xi$-coefficient in $(5.6 c)$ : the first one, $2 S K_{1} / k^{4}$, rapidly approaches zero as $S t$ increases (provided $K_{1} \neq 0$; if $K_{1}=0$, it vanishes), whereas the second one, $a_{0}^{2} /\left(S K_{1}\right)$, remains constant. Hence, if $S t$ is sufficiently large, the inequality $2 S K_{1} / k^{4} \ll a_{0}^{2} /\left(S K_{1}\right)$ holds, such that

$$
2 \frac{S^{2} \ell^{2}}{a_{0}^{2}} \ll\left[1+\frac{K_{3}^{2}}{K_{1}^{2}}+\frac{K_{2}^{2}}{K_{1}^{2}}-2 \frac{K_{2}}{K_{1}} S t+(S t)^{2}\right]^{2}\left(K_{1} \ell\right)^{2},
$$


where (4.2) has been used to express the magnitude of the time-dependent wavenumber in terms of its initial components, $K_{1}, K_{2}$ and $K_{3}$, and here $\ell$ is the lengthscale previously defined. Equation (5.7) is roughly equivalent to $M_{d} \ll(S t)^{2}$ for large $S t$, which defines the 'large-St' criterion as being $S t \gg M_{d}^{1 / 2}$ (assuming that the 'average' magnitude of the components of $\boldsymbol{K}$ that significantly contribute to the energy are of the order $1 / \ell$ ).

The 'crossover' behaviour cited above can be related to the change of regime caused by passing from small $S t$ - where $2 S K_{1} / k^{4}$ is relevant and can be dominant with respect to $a_{0}^{2} /\left(S K_{1}\right)$ at sufficiently large $M_{d}$ - to large $S t$, where $2 S K_{1} / k^{4}$ is never significant, even at large $M_{d}$. In the latter, even if the initial conditions are characterized by large $M_{d}$, the asymptotic state will be essentially independent of $M_{d}$, since the $2 S K_{1} / k^{4}$ term can be neglected in $(5.6 c)$, which can then be differentiated with respect to time to yield the simplified equation $\ddot{y}+a_{0}^{2} k^{2} y=0$. Although $k^{2}$ varies strongly with time in this equation, an oscillating (and thus bounded) solution for $y$ can be inferred using a WKB-type argument $\left(\omega=a_{0} k ; \dot{\omega} / \omega^{2}\right.$ becomes small at large $S t)$. Hence the behaviour at large $S t$ and that previously referred to as quasi-acoustic or quasi-solenoidal are similar. Considering the dynamic behaviour at sufficiently large $S t$, with solenoidal RDT one can compare the fixed points $(\dot{\xi}=\dot{y}=0)$ of the simplified system $(5.6 a-c)$ obtained by dropping the $2 S K_{1} / k^{4}$ term. We find

$$
\xi=\xi_{0}-S K_{1} z_{0}, \quad y=0, \quad z=0 .
$$

The corresponding RDT solution in the pure-solenoidal case is

$$
\xi=\xi_{0}, \quad y=0, \quad a_{0}^{2} z=a_{0}^{2} z^{s}=2 S \frac{K_{1}}{k^{4}} \xi_{0} .
$$

Note that $z=z^{s}$ is equivalent to the classic Poisson equation, as in (2.19)-(2.20), and $z^{s}$ is precisely the term that is neglected in (5.6c) at large $S t$. It is important to point out that $\xi_{0}=\xi(\boldsymbol{X}, 0)$ is an 'initial' condition chosen at any time for the pure-solenoidal case, whereas $\xi_{0}, z_{0}$ for the fixed-point solution (5.8) can be viewed as 'initial' data chosen at a time (after the crossover) for which the inequality (5.7) holds. The post-crossover state at intermediate $S t$ depends on the entire history, which is influenced by $M_{d}$ during the early history of the flow.

This semi-analytical analysis can explain the change of regime passing from small to large $S t$. It cannot, however, explain why the crossover occurs near $S t \approx 4$, for all $M_{d}$, and why, after the crossover, increasing $M_{d}$ causes the stabilization of the flow revealed by the RDT and DNS results. Two characteristics of the RDT equations can perhaps shed light on the matter:

(i) The first is the time-dependency of the wave vector, which physically reflects the kinematic distortion of a material bipoint (linear fluid element) $l_{i}(t)$ advected by the shear flow, in accordance with a wave-conservation law $k_{i} l_{i}=$ constant (see (2.14)). Consequently, the wavevector is primarily stretched (such that $|\boldsymbol{k}|$ increases) and its orientation becomes more and more vertical, due to the increase of $k_{2}$. This pureadvection effect tends to transfer energy toward smaller and smaller scales, which are less and less affected by compressibility (see e.g. figure 1 in Cambon et al. 1993 and the related discussion).

(ii) The second characteristic is the coupling term induced by the vortical part of the mean deformation (see $(2.12)$ and $(4.3 a, b)$ ). Even though the distortion of the wavevector also exists in an irrotational mean flow, this coupling term does not; this implies that it plays an essential role in the pure-shear crossover behaviour, although the coupling is less important at very small $S t$ (where the influence of the pure- 
straining irrotational part of the shear comes into play) and at very large $S t$, for the reasons given earlier in this section. Although for pure-shear flow the most important effect of compressibility (in the rapid-distortion regime) is to alter the structure of the solenoidal fluctuations, as reflected in the $M_{d}$-dependence of $b_{12}^{(s)}$ (figure $11 b$ ), the ultimate source of this alteration is the feedback of the dilatational disturbances upon the solenoidal field - since in a non-vortical mean flow the solenoidal fluctuations are completely independent of $M_{d}$, and are equivalent to those found in the $u_{i, i}=0$ case.

\subsubsection{Nonlinear and dissipative effects}

By comparing the RDT and DNS results presented above (see e.g. the $b_{12}$-histories in figure 9), one finds that much of the structural effect of compressibility is fairly well reproduced by the linear rapid-distortion analysis. This is especially true during the initial development of the flow, for a range of time that extends past that $(S t \approx 4)$ at which the destabilization-to-stabilization change in the role of compressibility occurs. At later times, however, as $S t \rightarrow \infty$, the DNS histories exhibit a dependence upon distortion Mach number that is not captured by the RDT. Although the asymptotic non-dimensional production $b_{12}$ predicted by the RDT appear to be converging to a unique value, independently of initial $M_{d}$ (figure $9 b$ ) (which is consistent with the simplified fixed-point analysis in §5.2.2), the same cannot be said for the full nonlinear solutions (figure 9a). Evidently, nonlinear and dissipative effects (the dissipative scales receiving energy through the nonlinear cascade) are not essential for predicting the general trends, although they are important in breaking the reversibility of linear solutions, causing a dependence on the compressibility parameter $M_{d}$ of the asymptotic results.

Some authors (e.g. Friedrich 1996) have recently pointed out that the dilatational dissipation tensor $\epsilon_{i j}^{d}$ is strongly anisotropic in shear flows, with the vertical component $\epsilon_{22}^{d}$ being dominant. While a major theme of this paper has been the irrelevance (compared to alteration of the turbulent kinetic energy production) of the rate of dilatational dissipation of turbulent kinetic energy $\epsilon_{d}=\frac{1}{2} \epsilon_{i i}^{d}$, one of the components (i.e. $\epsilon_{22}^{d}$ ) becoming dominant with respect to the others could lead to further modification of the Reynolds stress tensor, and thereby cause a further 'stabilizing' influence associated with compressibility. Although the RDT-based explanation of compressibility effects offered here and that attributed to anisotropy of the dilatational dissipation do not correspond, it is perhaps significant that both focus on the behaviour of the vertical (cross-gradient) components of the quantities of interest - be they velocity fluctuations, Reynolds stress tensors, or dissipation tensors. The recent DNS results analysed by Speziale et al. (1995) also suggest that a detailed comparison of the components of various anisotropy measures predicted by RDT and DNS might be a fruitful endeavour.

We are grateful to Professor G. A. Blaisdell and E. T. Spyropoulos for helpful suggestions and for allowing us to use their DNS code. Financial support for the first author was supplied by DRET (Direction des Recherches, Etudes et Techniques, DGA, French Defense), under contract 95/2585 A (Recherche fondamentale). Computational resources were made available on the Cray 98 by IDRIS (Institut de Développement et des Ressources en Informatique Scientifique), which receives major funding from the CNRS. 


\section{REFERENCES}

Batchelor, G. K. \& Proudman, I. 1954 The effect of rapid distortion of a fluid in turbulent motion. Q. J. Mech. Appl. Math. 7, 121-152.

BENOIT, J.P. 1992 Etude expérimentale et théorique d'une turbulence homogène soumise à des effets couplés de rotation et de déformation. Thèse de Doctorat, Université Claude Bernard Lyon I, France.

Blaisdell, G. A., Coleman, G. N. \& Mansour, N. N. 1996 Rapid distortion theory for compressible homogeneous turbulence under isotropic mean strain. Phys. Fluids 8, 2622-2705

Blaisdell, G. A., Mansour, N. N. \& Reynolds, W. C. 1991 Numerical simulation of compressible homogeneous turbulence. Dept of Mech. Engng, Stanford University, Thermosciences Div. Rep. TF-50.

Blaisdell, G. A., Mansour, N. N. \& Reynolds, W. C. 1993 Compressibility effects on the growth and structure of homogeneous turbulent shear flow. J. Fluid Mech. 256, 443-485.

Bradshaw, P. 1977 Compressible turbulent shear layers. Ann. Rev. Fluid Mech. 9, 33-54.

CAmbon, C. 1982 Etude spectrale d'un champ turbulent incompressible soumis à des effets couplés de déformation et de rotation imposés extérieurement. Thèse d'État, Université Claude Bernard Lyon I, France.

Cambon, C., Benoit, J. P., Shao, L. \& Jacquin, L. 1994 Stability analysis and large eddy simulation of rotating turbulence with organized eddies. J. Fluid Mech. 278, 175-200.

Cambon, C., Coleman, G. N. \& Mansour, N. N. 1993 Rapid distortion analysis and direct simulation of compressible homogeneous turbulence at finite Mach number. J. Fluid Mech. 257, 641-665.

Cambon, C., Teissedre, C. \& Jeandel, D. 1985 Etude d'effets couplés de déformation et de rotation sur une turbulence homogène. J. Méc. Théor. Appl. 4, 629-657

Clemens, N. T. \& Mungal, M. G. 1995 Large-scale structure and entrainment in the supersonic mixing layer. J. Fluid Mech. 284, 171-216.

Durbin, P. A. \& Zeman, O. 1992 Rapid distortion theory for homogeneous compressed turbulence with application to modelling. J. Fluid Mech. 242, 349-370.

ERINGEN, A. C. 1980 Mechanics of Continua. Wiley.

Fernholz, H. H. \& Finley, P. J. 1977 A critical compilation of compressible turbulent boundary layer data. AGARDograph 223.

Fernholz, H. H. \& Finley, P. J. 1980 A critical commentary on mean flow data for two-dimensional compressible turbulent boundary layers. AGARDograph 253.

Fernholz, H. H., Smits, A. J., Dussauge, J.-P. \& Finley, P. J. 1989 A survey of measurements and measuring techniques in rapidly distorted compressible turbulent boundary layers. AGARDograph 315 .

FRIEDRICH, R. 1993 Compressible turbulence. Lehrstuhl für Fluidmechanik, Technische Universität München, Space Course 1993 class notes.

Friedrich, R. 1996 Compressibility effects due to turbulent fluctuations. Eur. Turb. Conf. 6, Lausanne, 2-5 July 1996, Invited lecture.

Goldstein, M.E. 1978 Unsteady vortical and entropic distortions of potential flows round arbitrary obstacles. J. Fluid Mech. 89, 433-468.

Huang, P. G., Coleman, G. N. \& Bradshaw, P. 1995 Compressible turbulent channel flows: DNS results and modelling. J. Fluid Mech. 305, 185-218.

JACQuin, L., CAmbon, C. \& Blin, E. 1993 Turbulence amplification by a shock wave and rapid distortion theory. Phys. Fluids A 10, 2539-2550.

KeVlahan, N.K. R. \& Hunt, C. J. R. 1996 Nonlinear interactions in turbulence with strong irrotational straining. Submitted to J. Fluid Mech.

Kovasznay, L. S. G. 1953 Turbulence in supersonic flow. J. Aeronaut. Sci. 20, 657-682.

LeE, S., Lele, S. K. \& MoIN, P. 1993 Direct numerical simulation of isotropic turbulence interacting with a weak shock wave. J. Fluid Mech. 251, 533-562.

Lele, S. K. 1994 Compressibility effects on turbulence. Ann. Rev. Fluid Mech. 26, 211-254.

MARION, J.D. 1988 Etude spectrale d'une turbulence isotrope compressible. Thèse de Doctorat, Ecole Centrale de Lyon, France.

Monin, A. S. \& Yaglom, A. M. 1971 Statistical Fluid Mechanics: Mechanics of Turbulence. The MIT Press. 
Papamoschou, D. \& Roshko, A. 1988 The compressible turbulent mixing layer: an experimental study. J. Fluid Mech. 197, 453-477.

RogalLO, R. S. 1981 Numerical experiments in homogeneous turbulence. NASA TM-81315.

Rogers, M. M. 1991 The structure of a passive scalar field with a uniform mean gradient in rapidly sheared homogeneous turbulent flow. Phys. Fluids A 3, 144-154.

Salhi, A. \& CAMbON, C. 1996 A new analysis of rotating shear flows using linear approach and DNS-LES results. Submitted to J. Fluid Mech.

Sandham, N.D. \& Reynolds, W.C. 1991 Three-dimensional simulations of large eddies in the compressible mixing layer. J. Fluid Mech. 224, 133-158.

SARKAR, S. 1995 The stabilizing effect of compressibility in turbulent shear flow. J. Fluid Mech. 282, $163-186$.

SArkar, S., Erlebacher, G. \& Hussaini, M. Y. 1991 $a$ Direct simulation of compressible turbulence in a shear flow. Theoret. Comput. Fluid Dyn. 2, 291-305.

Sarkar, S., Erlebacher, G., Hussaini, M. Y. \& Kreiss, O. $1991 b$ The analysis and modelling of dilatational terms in compressible turbulence. J. Fluid Mech. 227, 473-493.

Settles, G. S. \& Dodson, L. J. 1991 Hypersonic shock/boundary layer interaction database. NASA Contractor Rep. 177577.

SimONE, A. 1995 Etude théorique et simulation numérique de la turbulence compressible en présence de cisaillement ou de variation de volume à grande échelle. Thèse de Doctorat, Ecole Centrale de Lyon, France.

Simone, A. \& CAmbon, C. 1995 a Revisiting compressible homogeneous shear flow at finite Mach number by means of rapid distortion theory. Proc. Fifth European Turbulence Conf. Siena, Italy. Advances in Turbulence (ed. R. Benzi). Kluwer.

Simone, A. \& CAmbon, C. $1995 b$ Rapid distortion and direct numerical approach to compressibility in turbulent shear flow. Tenth Symp. on Turbulent Shear Flows, Penn. State U., Aug. 14-16.

Speziale, C. G., Abid, R. \& Mansour, N. N. 1995 Evaluation of Reynolds stress turbulence closures in compressible homogeneous shear flow. J. Appl. Math. Phys., Special Issue 46, S717-S736.

Spina, E., Smits, A. J. \& Robinson, S. 1994 Supersonic turbulent boundary layers. Ann. Rev. Fluid Mech. 26, 287-319.

Spyropoulos, E. T. \& Blaisdell, G. A. 1996 Evaluation of the dynamic model for simulations of compressible decaying isotropic turbulence. AIAA J. 34, 990-998.

TAVOUlaris, S. \& CoRrsin, S. 1981 Experiments in nearly homogeneous turbulent shear flow with a uniform mean gradient temperature. Part I. J. Fluid Mech. 104, 311-347.

Townsend, A. A. 1976 The Structure of Homogeneous Turbulence. Cambridge University Press.

Vreman, W. A., Sandham, N.D. \& Luo, K.H. 1996 Compressible mixing layer growth rate and turbulence characteristics. J. Fluid Mech. 320, 235-258.

ZEMAN, O. 1990 Dilatation dissipation: The concept and application in modelling compressible mixing layers. Phys. Fluids A 2, 178-188. 